\title{
A new method for the preparation of $N$-stabilized allenylidene complexes of chromium and tungsten
}

\author{
Helmut Fischer*, Normen Szesni, Gerhard Roth, Nicolai Burzlaff, Bernhard Weibert \\ Fachbereich Chemie, Universität Konstanz, Fach M727, D-78457 Konstanz, Germany
}

Received 30 April 2003; received in revised form 5 June 2003; accepted 6 June 2003

\begin{abstract}
Displacement of tetrahydrofuran in $\left[(\mathrm{CO})_{5} \mathrm{M}(\mathrm{THF})\right](\mathrm{M}=\mathrm{Cr}, \mathrm{W})$ by the anion $[\mathrm{C} \equiv \mathrm{C}-\mathrm{C}(=\mathrm{X}) \mathrm{Y}]^{-}\left(\mathrm{X}=\mathrm{O} ; \mathrm{NR} ; \mathrm{Y}=\mathrm{NR}{ }_{2}^{\prime}, \mathrm{Ph}\right)$ followed by alkylation of the resulting metalate with $\left[\mathrm{R}_{3}^{\prime \prime} \mathrm{O}\right] \mathrm{BF}_{4}\left(\mathrm{R}^{\prime \prime}=\mathrm{Me}, \mathrm{Et}\right)$ offers a convenient and versatile route to $\pi$-donorsubstituted allenylidene complexes, $\left[(\mathrm{CO})_{5} \mathrm{M}=\mathrm{C}=\mathrm{C}=\mathrm{C}\left(\mathrm{XR}^{\prime \prime}\right) \mathrm{Y}\right]$. Allenylidene complexes in which the terminal carbon atom of the allenylidene ligand constitutes part of a heterocycle are likewise accessible by this reaction sequence. Reaction of $\left[(\mathrm{CO})_{5} \mathrm{M}(\mathrm{THF})\right]$ with $\mathrm{Li}[\mathrm{C} \equiv \mathrm{C}-\mathrm{C}(=\mathrm{NMe}) \mathrm{Ph}]^{-}$and subsequent protonation of the metalate afford $\left[(\mathrm{CO})_{5} \mathrm{M}=\mathrm{C}=\mathrm{C}=\mathrm{C}(\mathrm{NMeH}) \mathrm{Ph}\right]$ in high yield. As indicated by the spectroscopic data of the compounds and the X-ray analyses of three representative examples, these allenylidene complexes are best described as hybrids of allenylidene and zwitterionic alkynyl complexes with delocalisation of the electron pair at nitrogen towards the metal center. Dimethylamine reacts with the amino(phenyl)allenylidene $\operatorname{complex}\left[(\mathrm{CO})_{5} \mathrm{Cr}=\mathrm{C}=\mathrm{C}=\right.$ $\mathrm{C}\left(\mathrm{NMe}_{2}\right) \mathrm{Ph}$ ] (7a) by addition of the amine across the $\mathrm{C}_{\alpha}=\mathrm{C}_{\beta}$ bond to give selectively the $E$-alkenyl(amino)carbene complex $\left[(\mathrm{CO})_{5} \mathrm{Cr}=\mathrm{C}\left(\mathrm{NMe}_{2}\right)-\mathrm{CH}=\mathrm{C}\left(\mathrm{NMe}_{2}\right) \mathrm{Ph}\right](\mathbf{1 2})$. In contrast, the reaction of dimethylamine with the amino(methoxy)allenylidene complex $\left[(\mathrm{CO})_{5} \mathrm{Cr}=\mathrm{C}=\mathrm{C}=\mathrm{C}\left(\mathrm{NMe}_{2}\right) \mathrm{OMe}\right]$ (1a) proceeds by addition of the amine to the $\mathrm{C}_{\gamma}$ atom and subsequent elimination of methanol to give the substitution product $\left[(\mathrm{CO})_{5} \mathrm{Cr}=\mathrm{C}=\mathrm{C}=\mathrm{C}\left(\mathrm{NMe}_{2}\right)_{2}\right](\mathbf{1 3})$. Triphenylphosphane neither adds to the $\mathrm{C}_{\alpha}$ nor the $\mathrm{C}_{\gamma}$ atom of 7a but upon irradiation displaces a $\mathrm{CO}$ ligand to form a cis-allenylidene(tetracarbonyl)phosphane complex $\mathbf{1 5}$.
\end{abstract}

(C) 2003 Elsevier B.V. All rights reserved.

Keywords: Allenylidene complexes; Chromium; Tungsten; Propenylidene complexes; Carbene complexes

\section{Introduction}

Transition metal complexes containing metal-carbon double bonds $\left[\mathrm{M}=\mathrm{C}\left(\mathrm{sp}^{2}\right)\right.$ or $\left.\mathrm{M}=\mathrm{C}(\mathrm{sp})\right]$ are among the most powerful tools in modern organic and organometallic synthesis. Especially transition metal carbene complexes have found broad applications in synthesis and catalysis. In recent years metallacumulenes such as vinylidene complexes, $\left[\mathrm{L}_{n} \mathrm{M}=\mathrm{C}=\mathrm{C}\left(\mathrm{R}_{1}\right) \mathrm{R}_{2}\right]$, and allenylidene complexes, $\left[\mathrm{L}_{n} \mathrm{M}=\mathrm{C}=\mathrm{C}=\mathrm{C}\left(\mathrm{R}_{1}\right) \mathrm{R}_{2}\right]$, have attracted increasing interest [1]. A considerable number of theoretical and experimental studies confirm the great potential of these compounds for $\mathrm{C}-\mathrm{C}$ and $\mathrm{C}-\mathrm{X}$ bond formation [2-8]. Ruthenium allenylidene complexes have been proposed to be key intermediates in catalytic

\footnotetext{
* Corresponding author. Tel.: +49-7531-88-2783; fax: +49-753188-3136.

E-mail address: helmut.fischer@uni-konstanz.de (H. Fischer).
}

processes [9] and have found application in Diels-Alder reactions [10]. The presence of the linear unsaturated carbon chain makes metallacumulenes potentially useful as one dimensional molecular wires [11] and for optoelectronic applications [12].

The vast majority of allenylidene complexes are derived from 1,1-disubstituted propargylic alcohols bearing either alkyl or aryl groups at the terminal carbon atom [1]. In contrast, the number of heteroatom-substituted allenylidene complexes is rather scarce although two of the very first allenylidene complexes to be synthesized carried a dimethylamino substituent at the terminal carbon atom $[13,14]$. These complexes $\mathbf{B}$ were obtained by Fischer et al. from alkenyl(ethoxy)carbene complexes $\mathbf{A}$ by Lewis acid induced alcohol elimination (Scheme 1: $\mathrm{M}=\mathrm{Cr}, \mathrm{W} ; \mathrm{R}=\mathrm{Et} ; \mathrm{R}^{\prime}=\mathrm{Me}$; $\mathrm{R}^{\prime \prime}=\mathrm{Ph}$ ) [13]. Later on this method has been extended to the synthesis of some other amino stabilized allenylidene complexes [15]. Alkynyl carbene complexes C 


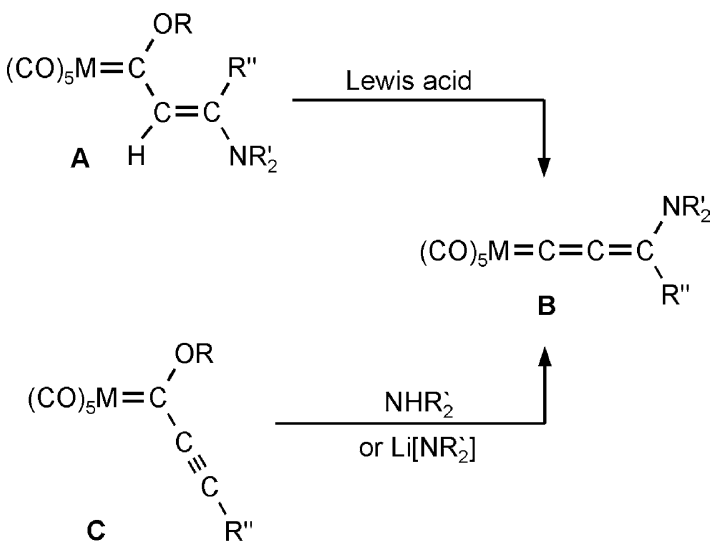

Scheme 1.

have also turned out to be useful precursors for the preparation of monoamino allenylidene complexes [16] (Scheme 1).

Recently a few cationic donor-substituted allenylidene complexes of ruthenium have been prepared by trapping of highly unstable butatrienylidene intermediate with amines [17] or by addition of amines to pentatetraenylidene complexes [17]. Two bis(dimethylamino)-substituted allenylidene complexes, $\left[(\mathrm{CO})_{5} \mathrm{M}=\mathrm{C}=\mathrm{C}=\right.$ $\mathrm{C}\left(\mathrm{NMe}_{2}\right)_{2}$ ] [18], and two alkenyl(dimetylamino) allenylidene complexes, $\left[(\mathrm{CO})_{5} \mathrm{M}=\mathrm{C}=\mathrm{C}=\mathrm{C}\left(\mathrm{NMe}_{2}\right)-\mathrm{CH}=\right.$ $\mathrm{C}\left(\mathrm{NMe}_{2}\right)_{2}$ ] $(\mathrm{M}=\mathrm{Cr}, \mathrm{W})$ [18], have also been synthesized.

The bonding situation in allenylidene complexes and their reactivity are expected to strongly depend on the substitution pattern. Therefore, we were looking for a new and broadly applicable approach to this type of complexes and now report on a simple one-step route for nitrogen-stabilized allenylidene complexes of chromium and tungsten and on the results of a few preliminary reactivity studies.

\section{Results and discussion}

\subsection{Synthesis}

Usually transition metal allenylidene complexes are prepared by introduction of a preformed $\mathrm{C}_{3}$ fragment into the coordination sphere of the metal. Thus, bis(aryl)- and bis(alkyl)-substituted allenylidene complexes are readily accessible by the reaction of 1,1disubstituted propargylic alcohols with a metal fragment followed by elimination of water [1]. By a related method bis(dimethylamino)allenylidene complexes of chromium and tungsten are synthesized. Reaction of $\left[(\mathrm{CO})_{5} \mathrm{M}(\mathrm{THF})\right]$ with the lithium salt of 3,3,3-tris(dimethylamino)prop-1-yne as the $\mathrm{C}_{3}$ source gives an alkynyl metalate and subsequent Lewis acid promoted elimination of dimethylamide [19]. These strategies cannot be applied to the synthesis of allenylidene complexes bearing two different heteroatom functionalities due to the lack of appropriate precursors.

This problem could be circumvented by using deprotonated propynoic acid amides as the $\mathrm{C}_{3}$ source. These amides readily reacted with pentacarbonyl(tetrahydrofuran)-chromium and -tungsten to form alkynyl metalates. Subsequent alkylation of the metalates by Meerwein salts and chromatography afforded the amino(alkoxy)-substituted allenylidene complexes $\mathbf{1 a}, \mathbf{b}-$ 6a,b in $42-80 \%$ isolated yield (Scheme 2).

This one-pot route is also applicable to the synthesis of amino(aryl)-substituted allenylidene complexes by employing the lithium salts of $C$-ethynylimines instead of deprotonated propynoic acid amides as the $\mathrm{C}_{3}$ source. For instance, deprotonation of methyl-(1-phenyl-prop2-ynylidene)-amine with butyllithium, followed by reaction of the acetylide with $\left[(\mathrm{CO})_{5} \mathrm{M}(\mathrm{THF})\right]$ and alkylation of the resulting metalate with oxonium salts, $\left[\mathrm{R}_{3}^{\prime} \mathrm{O}\right] \mathrm{BF}_{4}$, gave the dialkylamino(phenyl)allenylidene complexes $\mathbf{7 a , b}-\mathbf{8 a}, \mathbf{b}$ in $61-69 \%$ yield (Scheme 3 ). In addition to $\mathbf{7 a}, \mathbf{b}-\mathbf{8 a}, \mathbf{b}$, small amounts of the monoalkylamino(phenyl)allenylidene complexes $\mathbf{9 a}, \mathbf{b}$ were isolated as byproducts. The compounds $\mathbf{9 a}, \mathbf{b}$ presumably were formed by reaction of either the metalate with traces of water present in the solvent or of unreacted metalate with water adsorbed to $\mathrm{SiO}_{2}$ used as the stationary phase in the chromatography. In accord with this assumption, addition of $\mathrm{SiO}_{2}$ to solutions of the metalates gave $\mathbf{9 a}, \mathbf{b}$ as the single isolable product in 78 and $76 \%$ yield, respectively, (Scheme 3).

Up to now monoamino-substituted allenylidene complexes of Group 6 metals were only accessible by twostep methods, either from alkenyl(alkoxy)carbene com-
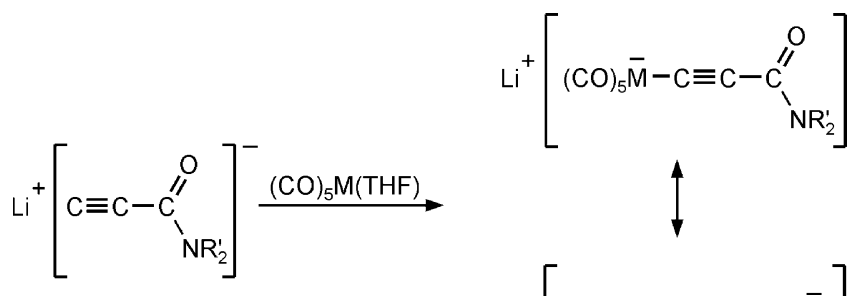

\begin{tabular}{c|rr}
$\mathrm{M}=\mathrm{Cr}(\mathbf{a}), \mathrm{W}(\mathbf{b})$ \\
$\mathrm{R}_{2}^{\prime}$ & $\mathrm{R}=\mathrm{Me}$ & $\mathrm{Et}$ \\
\hline $\mathrm{Me}_{2}$ & $\mathbf{1}$ & $\mathbf{2}$ \\
$-\left(\mathrm{CH}_{2}\right)_{4^{-}}$ & $\mathbf{3}$ & $\mathbf{4}$ \\
$\mathrm{Ph}_{2}$ & $\mathbf{5}$ & $\mathbf{6}$
\end{tabular}

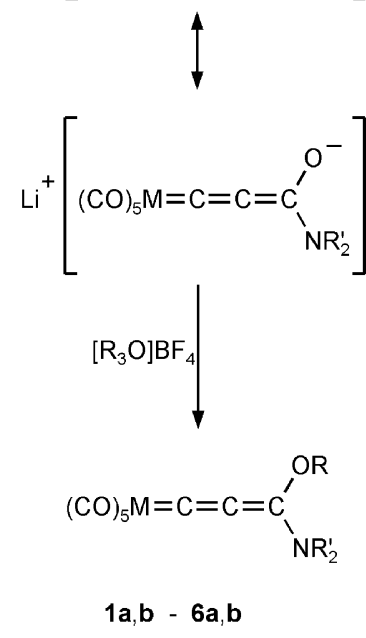

Scheme 2. 


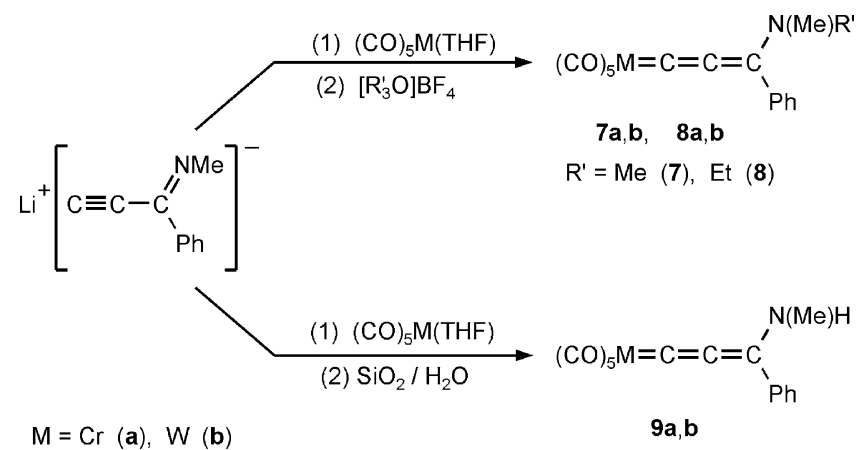

Scheme 3.

plexes via 1,2-elimination of an alcohol [13,15] or by treatment alkynylcarbene complexes with amines or amides (Scheme 1) [16]. In both cases $\alpha, \beta$-unsaturated carbene complexes have to be synthesized first.

When 2-ethynyl pyridine was used as the starting alkyne, allenylidene complexes $(\mathbf{1 0 a}, \mathbf{b}, \mathbf{1 1 a}, \mathbf{b})$ were obtained in which the terminal carbon atom of the chain forms part of an $N$-heterocycle (Scheme 4).

\subsection{Properties}

The new complexes were characterized by spectroscopic means and by elemental analyses. The IR spectra show a typical pentacarbonyl pattern in the region $1900-2100 \mathrm{~cm}^{-1}$ and additionally a $v(\mathrm{CCC})$ absorption between 1980 and $2010 \mathrm{~cm}^{-1}$. Within the series of complexes 1-11 the $v(\mathrm{CO})$ and $v(\mathrm{CCC})$ absorptions are almost independent of the nature of the substituents. Increasing the donor capacity of the allenylidene substituent results in only a slight shift of the $v(\mathrm{CCC})$ absorption band. In general the absorptions are at similar wave numbers than those of $\left[(\mathrm{CO})_{5} \mathrm{M}=\mathrm{C}=\mathrm{C}=\right.$ $\mathrm{C}\left(\mathrm{NMe}_{2}\right)_{2}$ ] [19]. From the position of the $v(\mathrm{CO})$ $A_{1}$ (trans) and $E$ vibrations at low energy and the $v(\mathrm{CCC})$ band at rather high energy it follows that these allenylidene ligands transfer considerable electron density to the metal center and that the polar resonance form III (Scheme 5) significantly contributes to the overall bonding description. In contrast, the $v(\mathrm{CO})$ $A_{1}$ (trans) and $E$ vibrations of bisaryl-substituted allenylidene complexes are found at considerably higher energy whereas their $A_{1}(c i s)-v(\mathrm{CO})$ and their $v(\mathrm{CCC})$ absorption are at lower energy indicating dominance of unpolar resonance forms similar to $\mathbf{I}[2 \mathrm{~b}, 20]$.

The conclusions drawn from the IR spectra concerning the importance of resonance form III of allenylidene complexes for their stabilization are supported by the NMR spectra:

(a) With increasing donor ability of the substituents at $\mathrm{C}_{\gamma}$ the ${ }^{13} \mathrm{C}$-NMR resonance of the metal-bound $\mathrm{C}_{\alpha}$ atom shifts towards higher field $\left(\mathrm{NPh}_{2} \rightarrow \mathrm{NMe}_{2} \rightarrow \mathrm{N}\left(\mathrm{CH}_{2}\right)_{4}\right.$ : $\Delta \delta$ ca. $11-15$ ppm, $\mathrm{Ph} \rightarrow \mathrm{OMe} \rightarrow \mathrm{NMe}_{2}: \Delta \delta$ ca. $40-58$ ppm).

(b) Two sets of ${ }^{1} \mathrm{H}-\mathrm{NMR}$ resonances for the $\mathrm{N}$-alkyl groups confirm the double bond character in the $\mathrm{C}_{\gamma}-\mathrm{N}$ bond.

(c) When solutions of $\mathbf{1}$ and $\mathbf{7}$ in $\left[d_{2}\right]$ tetrachloroethane were heated up to $120^{\circ} \mathrm{C}$ no coalescence of the signals was observed. Therefore the rotational barrier of allenylidene complexes bearing one amino substituent is higher than $19 \mathrm{kcal} \mathrm{mol}^{-1}$. Contrary to $\mathbf{1 - 9}$, the bis(amino)allenylidene complexes $\left[(\mathrm{CO})_{5} \mathrm{M}=\mathrm{C}=\mathrm{C}=\right.$ $\mathrm{C}\left(\mathrm{NMe}_{2}\right)_{2}$ ] exhibit only one $\mathrm{N}$-Me resonance which neither splits nor broadens on cooling solutions down to $-90{ }^{\circ} \mathrm{C}[19]$.

The NMR spectra of the complexes 8 indicate the presence of two isomers $(\boldsymbol{E}-\mathbf{8}: \boldsymbol{Z}-\mathbf{8}$ ratio $=2: 3)$ differing in the relative orientation of the $N$-alkyl groups (Scheme 6). As expected on the basis of the NMR-spectroscopic results with 1 and 7, the ratio of these conformers does not change upon warming. These rotational conformers exhibit strong shift differences not only for the $\mathrm{N}$-alkyl groups but also for the chain carbon atoms and the trans-CO carbon atom. Analogously to $\mathbf{8}$, the formation of two isomers in the synthesis of a comparable indolinyl-substituted allenylidene complex was reported earlier [15]. Due to the considerable differences in size of the $N$-bound groups in $9(\mathrm{Me}$ and $\mathrm{H})$, this compound selectively adopts the $Z$-conformation.

As expected from the polar resonance forms II and III (Scheme 5) the compounds 1-11 exhibit a significant negative solvatochromic effect. The UV-vis absorption at lowest energy which is assigned to a MLCT transition shifts toward shorter wavelengths when unpolar or weakly polar solvents are replaced by more polar ones. The effect is most pronounced with the complexes $\mathbf{9 , 1 0}$ and 11 in which an optimal $\pi$-interaction of the lone electron pair at nitrogen with the chain is ensured either

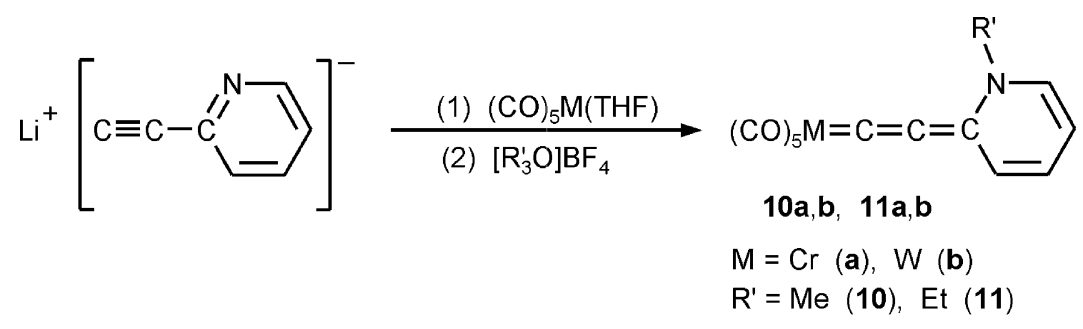

Scheme 4. 


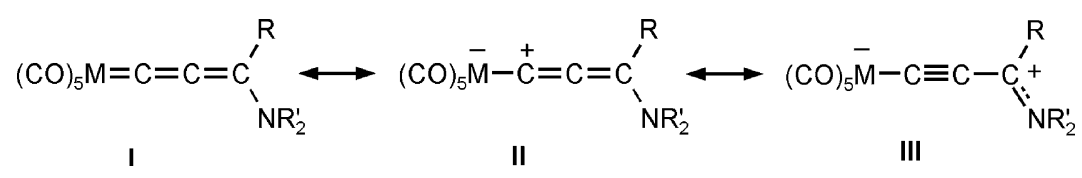

Scheme 5 .

by incorporation of the nitrogen atom into a planar ring (as in $\mathbf{1 0}$ and 11) or by small substitutents at nitrogen (Me and $\mathrm{H}$ in 9).

The solid-state structures of complexes $\mathbf{1 b}, \mathbf{7 b}$ and 11a were additionally established by X-ray structural analyses (Figs. 1-3, Tables 1 and 2). The allenylidene backbones deviate only slightly from linearity. The individual bond lengths along the $\mathrm{MC}_{3}$ fragment differ markedly, however, agree well with those of other monoamino-substituted allenylidene complexes [16c, 16f]. The $\mathrm{C} 6-\mathrm{C} 7$ bond is short $(1.225-1.253 \AA)$ and resembles an elongated $\mathrm{CC}$ triple bond whereas the $\mathrm{C} 7-$ C8 bond is rather long [1.399(8)-1.409(2) $\mathrm{A}]$ when compared to a $\mathrm{C}(\mathrm{sp})=\mathrm{C}\left(\mathrm{sp}^{2}\right)$ bond (e.g. $1.340 \AA$ in $\operatorname{Mes}(\mathrm{Ph}) \mathrm{C}=\mathrm{C}=\mathrm{C}(\mathrm{Ph}) \mathrm{Mes}[21])$. The difference in bond length $d(\mathrm{C} 6-\mathrm{C} 7)-d(\mathrm{C} 7-\mathrm{C} 8)$ in these complexes is significantly more pronounced than in the diphenylsubstituted allenylidene complex $\left[(\mathrm{CO})_{5} \mathrm{Cr}=\mathrm{C}=\mathrm{C}=\mathrm{CPh}_{2}\right]$ [20]. The atoms $\mathrm{C} 8$ and $\mathrm{N} 1$ are planar coordinated (sums of angles: $360^{\circ} \mathrm{C}$ ). Together with the short $\mathrm{C} 8-$ $\mathrm{N} 1$ bond, this confirms the delocalisation of the electron pair at nitrogen towards the metal center. The short trans-CO (M1-C5) bond in 1b and 11a also illustrates the strong donor ability of the terminal substituents. Like in 1,2-dihydropyridinylidene complexes [22] the dihydropyridinyl ring in 11a is essentially planar.

\subsection{Reactions}

A theoretical study predicts that hard nucleophiles like amines will attack allenylidene(pentacarbonyl) complexes preferentially at the $\alpha$ carbon atom [2a]. This is confirmed by experimental observations. Monoaminosubstituted allenylidene complexes are known to add dimethylamine exclusively to the $\mathrm{C}_{\alpha}-\mathrm{C}_{\beta}$ bond giving 1,3-bis(amino)propenylidene complexes [16c]. Nonhet-

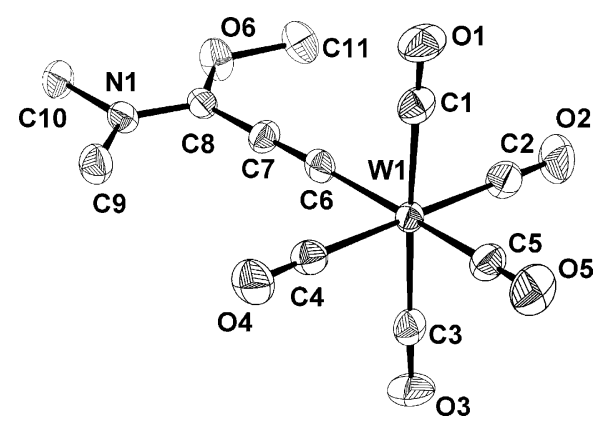

Fig. 1. Structure of complex $\mathbf{1 b}$ in the crystal (ellipsoids drawn at 50\% level, hydrogens omitted for clarity).

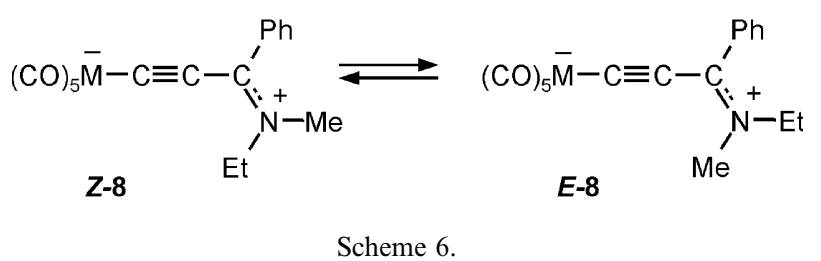

eroatom-substituted allenylidene complexes usually show the same reactivity pattern towards amines [3a]. However, by NMR spectroscopy a $\mathrm{C}_{\gamma}$ adduct was shown to be a transient intermediate in the reaction of [triphos $\left.(\mathrm{CO})_{2} \mathrm{Re}=\mathrm{C}=\mathrm{C}=\mathrm{CPh}_{2}\right]^{+}$with $\mathrm{NH}_{3}$ to form the $\mathrm{C}_{\alpha}-\mathrm{C}_{\beta}$ adduct [10].

Analogously and as expected, the reaction of complex 7a with dimethylamine at room temperature yields quantitatively carbene complex $\mathbf{1 2}$. Only the formation of the $E$-configuration is detected. In principle, the addition constitutes the reverse of the first synthesis of aminoallenylidene complexes (see Scheme 1: $\mathbf{A} \rightarrow \mathbf{B}$ ) [13].

The reaction of the amino(alkoxy)-substituted complex 1a with four equivalents of dimethylamine strongly deviates from this reaction pattern. Instead of addition across the $\mathrm{C}_{\alpha}-\mathrm{C}_{\beta}$ bond, the substitution of the alkoxy group of the allenylidene ligand to form the bis(dimethylamino)allenylidene complex $\mathbf{1 3}$ (isolated yield: $11 \%$ ) as well as substitution of the complete allenylidene ligand to give the dimethylamino complex 14 [23] in 9\% yield is observed (Scheme 7). Apparently, compound $\mathbf{1 3}$ is derived from a primary $\mathrm{C}_{\gamma}$ adduct with subsequent elimination of methanol. The substitution of a terminal group in allenylidene(carbonyl) complexes has not been observed before. Therefore, not only the nature of the nucleophile but also the substituents at the allenylidene complex play an important role in determining the regioselectivity of the addition. In contrast to $7 \mathbf{a}$,

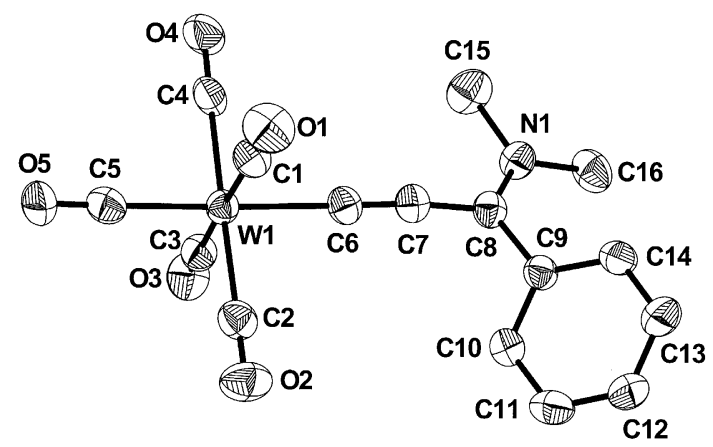

Fig. 2. Structure of complex $\mathbf{7 b}$ in the crystal (ellipsoids drawn at $50 \%$ level, hydrogens omitted for clarity). 
Table 1

Selected bond distances $(\AA)$ and angles $\left(^{\circ}\right)$ in $\mathbf{1 b}, \mathbf{7 b}$, and 11a

\begin{tabular}{llll}
\hline & $\mathbf{1 b}(\mathrm{M}=\mathrm{W})$ & $\mathbf{7 b}(\mathrm{M}=\mathrm{W})$ & $\mathbf{1 1 a}(\mathrm{M}=\mathrm{Cr})$ \\
\hline Bond length & & & \\
M1-C1 & $2.050(10)$ & $2.032(7)$ & $1.909(2)$ \\
M1-C2 & $2.053(10)$ & $2.072(7)$ & $1.916(2)$ \\
M1-C3 & $2.029(10)$ & $2.058(7)$ & $1.903(2)$ \\
M1-C4 & $2.063(10)$ & $2.036(6)$ & $1.902(2)$ \\
M1-C5 & $1.979(11)$ & $2.018(7)$ & $1.879(2)$ \\
M1-C6 & $2.142(10)$ & $2.150(6)$ & $2.042(2)$ \\
C6-C7 & $1.253(13)$ & $1.228(8)$ & $1.225(2)$ \\
C7-C8 & $1.408(12)$ & $1.399(8)$ & $1.409(2)$ \\
C8-N1 & $1.334(10)$ & $1.328(7)$ & $1.381(2)$ \\
C8-O6 & $1.289(10)$ & & \\
C8-C9 & & $1.488(7)$ & $1.412(2)$ \\
Bond angles & & & \\
M1-C6-C7 & $176.7(7)$ & $175.3(5)$ & $176.9(2)$ \\
C6-C7-C8 & $179.5(9)$ & $174.0(6)$ & $175.0(2)$ \\
C7-C8-N1 & $119.8(7)$ & $121.9(5)$ & $119.9(2)$ \\
C7-C8-O6 & $124.0(7)$ & & \\
C7-C8-C9 & & $116.6(5)$ & $122.8(2)$ \\
\hline
\end{tabular}

complex 13 does not add dimethylamine to form a carbene complex similar to $\mathbf{1 2}$ as already reported [19].

Tertiary phosphanes were found to add either to the $\mathrm{C}_{\alpha}$ or the $\mathrm{C}_{\gamma}$ allenylidene atom [2,3b, 23-26]. When $\mathrm{C}_{\gamma}$

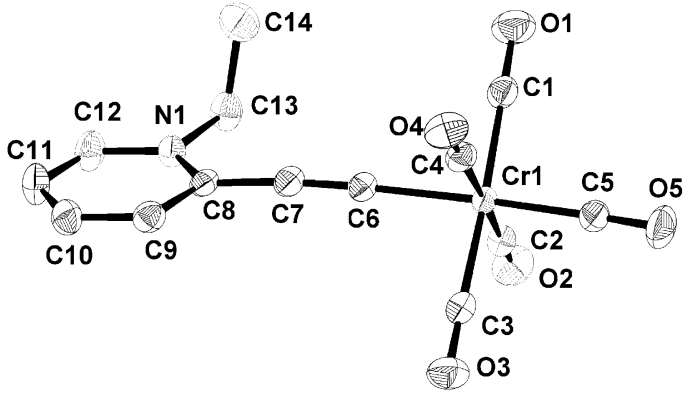

Fig. 3. Structure of complex 11a in the crystal (ellipsoids drawn at $50 \%$ level, hydrogens omitted for clarity).

adducts are initially formed, they slowly isomerize to give the $\mathrm{C}_{\alpha}$ phosphane adducts. When secondary phosphanes are employed in the reactions with diarylallenylidene complexes, the $\mathrm{C}_{\alpha}$ adducts rearrange by insertion of the phosphane into the metal $-\mathrm{C}_{\alpha}$ bond affording various types of phosphane complexes [26].

Deviating from these reactivity pattern, the aminostabilized allenylidene complexes do not thermally react with triphenylphosphane. Upon irradiation, the phosphane displaces one $\mathrm{CO}$ ligand in $7 \mathbf{a}$ yielding the allenylidene(tetracarbonyl)triphenylphosphane complex

Table 2

Crystal data and refinement details for compounds $\mathbf{1 b}, \mathbf{7 b}$ and $\mathbf{1 1 a}$

\begin{tabular}{|c|c|c|c|}
\hline & $1 \mathrm{~b}$ & $7 \mathrm{~b}$ & $11 \mathrm{a}$ \\
\hline Formula & $\mathrm{C}_{11} \mathrm{H}_{9} \mathrm{NO}_{6} \mathrm{~W}$ & $\mathrm{C}_{16} \mathrm{H}_{11} \mathrm{NO}_{5} \mathrm{~W}$ & $\mathrm{C}_{14} \mathrm{H}_{9} \mathrm{CrNO}_{5}$ \\
\hline$M_{\mathrm{r}}$ & 435.04 & 481.11 & 323.22 \\
\hline Crystal system & Monoclinic & Monoclinic & Monoclinic \\
\hline Space group & $P 2(1) / c$ & $P 2(1) / c$ & $C 2 / c$ \\
\hline$a(\AA)$ & $10.837(5)$ & $7.323(4)$ & $15.908(7)$ \\
\hline$b(\AA)$ & $12.577(4)$ & $21.633(11)$ & $9.854(4)$ \\
\hline$c(\AA)$ & $10.465(4)$ & $10.764(6)$ & $19.035(8)$ \\
\hline$\alpha\left({ }^{\circ}\right)$ & 90 & 90 & 90 \\
\hline$\beta\left({ }^{\circ}\right)$ & $107.09(3)$ & $103.43(5)$ & $104.63(3)$ \\
\hline$\gamma\left({ }^{\circ}\right)$ & 90 & 90 & 90 \\
\hline$V\left(\AA^{3}\right)$ & $1363.3(9)$ & $1658.5(15)$ & $2887.0(2)$ \\
\hline$Z$ & 4 & 4 & 8 \\
\hline Crystal size $\left(\mathrm{mm}^{3}\right)$ & $0.25 \times 0.20 \times 0.10$ & $0.50 \times 0.40 \times 0.30$ & $0.50 \times 0.45 \times 0.40$ \\
\hline$\rho_{\text {calc }}\left(\mathrm{g} \mathrm{cm}^{-3}\right)$ & 2.120 & 1.927 & 1.487 \\
\hline$\mu\left(\mathrm{mm}^{-1}\right)$ & 8.493 & 6.988 & 0.812 \\
\hline$F\left(\begin{array}{lll}0 & 0 & 0\end{array}\right)$ & 816 & 912 & 1312 \\
\hline Diffractometer & Siemens P4 & Siemens P4 & Siemens P4 \\
\hline Radiation & $\mathrm{Mo}-\mathrm{K}_{\alpha}$ & $\mathrm{Mo}-\mathrm{K}_{\alpha}$ & $\mathrm{Mo}-\mathrm{K}_{\alpha}$ \\
\hline$\lambda(\AA)$ & 0.71073 & 0.71073 & 0.71073 \\
\hline$T(\mathrm{~K})$ & $188(2)$ & $183(2)$ & $188(2)$ \\
\hline $\operatorname{Max} .2 \Theta\left({ }^{\circ}\right)$ & 54 & 54 & 56 \\
\hline Index range & $\begin{array}{l}-13 \leq h \leq 13 \\
-16 \leq k \leq 9 \\
-13 \leq l \leq 13\end{array}$ & $\begin{array}{l}-1 \leq h \leq 9 \\
-1 \leq k \leq 27 \\
-13 \leq l \leq 13\end{array}$ & $\begin{array}{l}-20 \leq h \leq 20 \\
-13 \leq k \leq 13 \\
-25 \leq l \leq 25\end{array}$ \\
\hline No. of data & 4396 & 4715 & 6728 \\
\hline No. of unique data & 2967 & 3630 & 3489 \\
\hline Parameters & 173 & 208 & 191 \\
\hline$R(F)$ for $I>2 \sigma(I)$ & 0.0460 & 0.0388 & 0.0364 \\
\hline$w R_{2}\left(F^{2}\right)$ for all data & 0.1307 & 0.1060 & 0.1004 \\
\hline Goodness-of-fit on $F^{2}$ & 1.185 & 1.115 & 1.061 \\
\hline
\end{tabular}




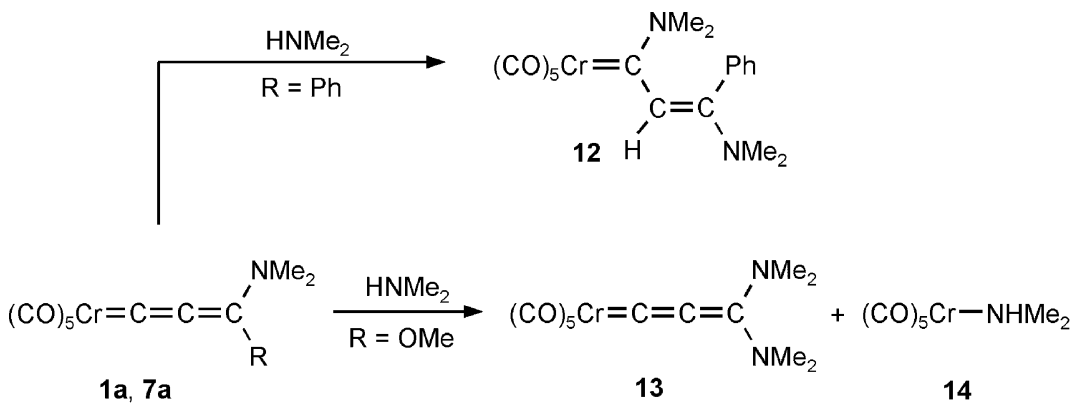

Scheme 7.

15 (Scheme 8). The IR absorptions show the typical pattern of cis-tetracarbonyl phosphane complexes. The trans-isomer is not observed under these reaction conditions. It also is not formed when a solution of $\mathbf{1 5}$ in toluene is heated to $80{ }^{\circ} \mathrm{C}$ for $4 \mathrm{~h}$. The ${ }^{13} \mathrm{C}$-NMR resonances of the allenylidene chain in $\mathbf{1 5}$ differ slightly from those of the pentacarbonyl complex 7a. With respect to $7 \mathbf{a}$, the $\mathrm{C}_{\alpha}$ and $\mathrm{C}_{\gamma}$ resonances are shifted downfield whereas the $C_{\beta}$ signal is upfield. These data indicate that the replacement of one acceptor $(\mathrm{CO})$ by the donor ligand phosphane gives rise to a reduction of the electron transfer from the amino group to the metal center.

In summary, the one-pot reaction sequence of $\left[(\mathrm{CO})_{5} \mathrm{M}(\mathrm{THF})\right]$ with alkynyl lithium salts of the type $\mathrm{Li}[\mathrm{C} \equiv \mathrm{C}-\mathrm{C}(=\mathrm{X}) \mathrm{Y}]$ and alkylation offers a convenient and versatile route to various $\pi$-donor-substituted allenylidene complexes. Our preliminary studies indicate that the reactivity of these compounds deviates from that of the corresponding diaryl- and dialkyl-substituted allenylidene complexes.

\section{Experimental}

\subsection{General}

All operations were performed in an inert gas atmosphere using standard Schlenk techniques. Solvents were dried by distillation from $\mathrm{CaH}_{2} \cdot\left(\mathrm{CH}_{2} \mathrm{Cl}_{2}\right), \mathrm{LiAlH}_{4}$ (pentane, $\mathrm{Et}_{2} \mathrm{O}$ ) and sodium (THF). The silica gel used for chromatography (Baker, silica gel for flash chromatography) was argon saturated. The reported yields refer to analytically pure substances and are not optimized. Instrumentation: ${ }^{1} \mathrm{H}-\mathrm{NMR},{ }^{13} \mathrm{C}-\mathrm{NMR}$, and ${ }^{31} \mathrm{P}-\mathrm{NMR}$

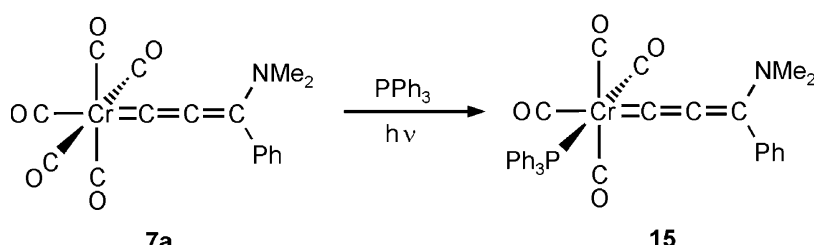

Scheme 8. spectra were recorded with a JEOL JNX 400 spectrometer. Chemical shifts are reported relative to the residual solvent peaks $\left({ }^{1} \mathrm{H},{ }^{13} \mathrm{C}\right)$ or $100 \% \mathrm{H}_{3} \mathrm{PO}_{4}\left({ }^{31} \mathrm{P}\right)$. IR: Biorad FTS 60. UV-vis: Hewlett-Packard diode array spectrophotometer 8453. MS: Finnigan MAT 312. Elemental analysis: Heraeus CHN-O-Rapid. The following alkynes were prepared according to literature procedures: Propynoic acid dimethylamide [27], 1-pyrrolidin-1-yl-propynone [28], propynoic acid diphenylamide [29], methyl(1-phenyl-prop-2-ynylidene)amine [30].

\subsection{General procedure for the preparation of the allenylidene complexes 1-11}

A solution of $3.1 \mathrm{ml}$ of $n$ - BuLi ( $5 \mathrm{mmol}, 1.6 \mathrm{M}$ in $n$ hexane) was added at $-80^{\circ} \mathrm{C}$ dropwise to a solution of $5 \mathrm{mmol}$ of the appropriate alkyne in $50 \mathrm{ml}$ of dry THF. The solution was stirred for $20 \mathrm{~min}$ at this temperature and then $50 \mathrm{ml}$ of a solution of $\left[\left(\mathrm{CO}_{5} \mathrm{M}(\mathrm{THF})\right](0.1 \mathrm{M}\right.$ in THF) was added. The cooling bath was removed and the brown solution was stirred for $30 \mathrm{~min}$ at ambient temperature. The solvent was removed in vacuo. The remaining oily residue was dissolved in $50 \mathrm{ml}$ of $\mathrm{CH}_{2} \mathrm{Cl}_{2}$ and treated with $5 \mathrm{mmol}$ of $\left[\mathrm{R}_{3} \mathrm{O}\right] \mathrm{BF}_{4}$ at $0{ }^{\circ} \mathrm{C}$ and stirred for further $60 \mathrm{~min}$ at this temperature. The complexes $7 \mathbf{a}$ and $7 \mathbf{b}$ were prepared by adding $10 \mathrm{~g}$ of argon-saturated silica gel to the solution of the metalate. The resulting deeply colored solution was filtered through a $5 \mathrm{~cm}$ layer of silica gel at $-20{ }^{\circ} \mathrm{C}$ using $\mathrm{CH}_{2} \mathrm{Cl}_{2}$ as eluent. The solvent was removed in vacuo and the residue was chromatographed on silica gel at $-20{ }^{\circ} \mathrm{C}$ using mixtures of pentane $/ \mathrm{CH}_{2} \mathrm{Cl}_{2}$ as the eluent.

\subsubsection{Pentacarbonyl(3-dimethylamino-3-methoxy-1,2- propadienylidene) chromium (1a)}

Yellow solid. Yield: $0.71 \mathrm{~g}(47 \%)$. M.p. $134-135^{\circ} \mathrm{C}$. IR (THF, $\left.\mathrm{cm}^{-1}\right): v(\mathrm{CO})=2080 \mathrm{vw}, 1933 \mathrm{vs}, 1912 \mathrm{~m}$; $v(\mathrm{CCC})=2008 \mathrm{~m} .{ }^{1} \mathrm{H}-\mathrm{NMR}\left(400 \mathrm{MHz}, \mathrm{CDCl}_{3}\right): \delta=$ $3.14\left(\mathrm{~s}, 3 \mathrm{H}, \mathrm{NCH}_{3}\right), 3.47\left(\mathrm{~s}, 3 \mathrm{H}, \mathrm{NCH}_{3}\right), 4.22(\mathrm{~s}, 3 \mathrm{H}$, $\left.\mathrm{OCH}_{3}\right) .{ }^{13} \mathrm{C}-\mathrm{NMR}\left(100.5 \mathrm{MHz}, \mathrm{CDCl}_{3}\right): \delta=36.7,41.7$ $\left(\mathrm{NCH}_{3}\right), 60.4\left(\mathrm{OCH}_{3}\right), 101.9\left(\mathrm{C}_{\beta}\right), 148.4\left(\mathrm{C}_{\gamma}\right), 209.5\left(\mathrm{C}_{\alpha}\right)$, 217.6 (cis-CO), 221.7 (trans-CO). MS (EI), $m / z$ (\%): 303 (14) $\left[\mathrm{M}^{+}\right], 219$ (6) $\left[\mathrm{M}^{+}-3 \mathrm{CO}\right], 191$ (19) $\left[\mathrm{M}^{+}{ }_{-} 4 \mathrm{CO}\right]$, 
$163(100)\left[\left(\mathrm{M}^{+}-5 \mathrm{CO}\right] . \mathrm{UV}-\mathrm{vis}: \lambda_{\max }(\log \varepsilon)\right.$ [solvent]: $432 \mathrm{~nm}$ (3.835) [pentane]; $410 \mathrm{~nm}$ (4.247) $\left[\mathrm{CHCl}_{3}\right] ; 400$ $\mathrm{nm}$ (4.223) $\left[\mathrm{CH}_{2} \mathrm{Cl}_{2}\right] ; 382 \mathrm{~nm}$ (4.125) [DMF]. Anal. Found: $\mathrm{C}, 43.46 ; \mathrm{H}, 3.09 ; \mathrm{N}, 4.64$. Calc. for $\mathrm{C}_{11} \mathrm{H}_{9} \mathrm{NO}_{6} \mathrm{Cr}$ (303.19): C, 43.58; H, 2.99; N, 4.62\%.

\subsubsection{Pentacarbonyl(3-dimethylamino-3-methoxy-1,2- propadienylidene)tungsten (1b)}

Yellow solid. Yield: $1.22 \mathrm{~g}(56 \%)$. M.p. $158-160{ }^{\circ} \mathrm{C}$ (dec.). IR (THF, $\mathrm{cm}^{-1}$ ): $v(\mathrm{CO})=2084 \mathrm{vw}, 1928 \mathrm{vs,}$ $1905 \mathrm{~m} ; \quad v(\mathrm{CCC})=2010 \mathrm{~m} .{ }^{1} \mathrm{H}-\mathrm{NMR} \quad(400 \mathrm{MHz}$, $\left.\mathrm{CDCl}_{3}\right): \delta=3.15\left(\mathrm{~s}, 3 \mathrm{H}, \mathrm{NCH}_{3}\right), 3.47\left(\mathrm{~s}, 3 \mathrm{H}, \mathrm{NCH}_{3}\right)$, $4.23\left(\mathrm{~s}, 3 \mathrm{H}, \mathrm{OCH}_{3}\right) .{ }^{13} \mathrm{C}-\mathrm{NMR}\left(100.5 \mathrm{MHz}, \mathrm{CDCl}_{3}\right)$ : $\delta=37.0,41.9\left(\mathrm{NCH}_{3}\right), 60.8\left(\mathrm{OCH}_{3}\right), 100.7\left({ }^{2} J_{\mathrm{WC}}=24.5\right.$ $\left.\mathrm{Hz}, \mathrm{C}_{\beta}\right), 150.4\left(\mathrm{C}_{\gamma}\right), 188.1\left(J_{\mathrm{WC}}=102.5 \mathrm{~Hz}, \mathrm{C}_{\alpha}\right), 197.0$ $\left(J_{\mathrm{WC}}=124.5 \mathrm{~Hz}\right.$, cis $\left.-\mathrm{CO}\right), 201.4 \quad\left(J_{\mathrm{WC}}=130.7 \mathrm{~Hz}\right.$, trans-CO). MS (EI), m/z (\%): 435 (69) $\left[\mathrm{M}^{+}\right], 407$ (45) [( $\left.\mathrm{M}^{+}-\mathrm{CO}\right], 379(93)\left[\mathrm{M}^{+}-2 \mathrm{CO}\right], 349(30)\left[\mathrm{M}^{+}-3 \mathrm{CO}\right]$, 323 (44) $\left[\mathrm{M}^{+}-4 \mathrm{CO}\right], 295$ (100) $\left[\mathrm{M}^{+}{ }_{-5 \mathrm{CO}}\right], 250$ (59) $\left[\mathrm{M}^{+}-5 \mathrm{CO}-3 \mathrm{Me}\right], 236(42)\left[(\mathrm{WCCCO})^{+}\right], 208$ (39) $\left[(\mathrm{WCC})^{+}\right] . \quad \mathrm{UV}-$ vis: $\lambda_{\max }(\log \varepsilon)$ [solvent]: $430 \mathrm{~nm}$ (4.237) [pentane]; $410 \mathrm{~nm}$ (4.293) $\left[\mathrm{CHCl}_{3}\right] ; 400 \mathrm{~nm}$ (4.212) $\left[\mathrm{CH}_{2} \mathrm{Cl}_{2}\right] ; 378 \mathrm{~nm}$ (4.075) [DMF]. Anal. Found: $\mathrm{C}, 30.54 ; \mathrm{H}, 2.22 ; \mathrm{N}, 3.40$. Calc. for $\mathrm{C}_{11} \mathrm{H}_{9} \mathrm{NO}_{6} \mathrm{~W}$ (435.05): C, 30.37; H, 2.09; N, 3.22\%.

\subsubsection{Pentacarbonyl(3-dimethylamino-3-ethoxy-1,2- propadienylidene) chromium (2a)}

Yellow solid. Yield: $0.86 \mathrm{~g}(54 \%)$. M.p. $82-84{ }^{\circ} \mathrm{C}$. IR $\left(\mathrm{THF}, \mathrm{cm}^{-1}\right): v(\mathrm{CO})=2080$ vw, 1932 vs, $1911 \mathrm{~m}$; $v(\mathrm{CCC})=2008 \mathrm{~m} .{ }^{1} \mathrm{H}-\mathrm{NMR}\left(400 \mathrm{MHz}, \mathrm{CDCl}_{3}\right): \delta=$ $1.45\left(\mathrm{t},{ }^{3} J_{\mathrm{HH}}=7.1 \mathrm{~Hz}, 3 \mathrm{H}, \mathrm{OCH}_{2} \mathrm{CH}_{3}\right), 3.14(\mathrm{~s}, 3 \mathrm{H}$, $\left.\mathrm{NCH}_{3}\right), 3.47\left(\mathrm{~s}, 3 \mathrm{H}, \mathrm{NCH}_{3}\right), 4.67\left(\mathrm{q},{ }^{3} J_{\mathrm{HH}}=7.1 \mathrm{~Hz}, 3 \mathrm{H}\right.$, $\left.\mathrm{OCH}_{2} \mathrm{CH}_{3}\right) .{ }^{13} \mathrm{C}-\mathrm{NMR}\left(100.5 \mathrm{MHz}, \mathrm{CDCl}_{3}\right): \delta=14.5$ $\left(\mathrm{OCH}_{2} \mathrm{CH}_{3}\right), 36.7,41.5\left(\mathrm{NCH}_{3}\right), 70.6\left(\mathrm{OCH}_{2} \mathrm{CH}_{3}\right)$, $101.9\left(\mathrm{C}_{\beta}\right), 147.9\left(\mathrm{C}_{\gamma}\right), 207.4\left(\mathrm{C}_{\alpha}\right), 217.6$ (cis-CO), 221.7 (trans-CO). MS (EI), m/z (\%): 317 (12) $\left[\mathrm{M}^{+}\right]$, 205 (18) $\left[\mathrm{M}^{+}-4 \mathrm{CO}\right], 177$ (100) $\left[\mathrm{M}^{+}-5 \mathrm{CO}\right]$. UV-vis: $\lambda_{\max }(\log \varepsilon)$ [solvent]: $430 \mathrm{~nm}$ (4.302) [pentane]; $406 \mathrm{~nm}$ (4.249) $\left[\mathrm{CHCl}_{3}\right] ; 398 \mathrm{~nm}$ (4.216) $\left[\mathrm{CH}_{2} \mathrm{Cl}_{2}\right] ; 380 \mathrm{~nm}$ (4.119) [DMF]. Anal. Found: $\mathrm{C}, 45.68 ; \mathrm{H}, 3.55 ; \mathrm{N}$, 4.51. Calc. for $\mathrm{C}_{12} \mathrm{H}_{11} \mathrm{NO}_{6} \mathrm{Cr}$ (317.22): $\mathrm{C}$, 45.44; $\mathrm{H}, 3.50$; $\mathrm{N}, 4.42 \%$.

\subsubsection{Pentacarbonyl(3-dimethylamino-3-ethoxy-1,2- propadienylidene) tungsten $(\mathbf{2} \boldsymbol{b})$}

Yellow solid. Yield: $1.38 \mathrm{~g}(62 \%)$. M.p. $100-101{ }^{\circ} \mathrm{C}$. IR (THF, $\left.\mathrm{cm}^{-1}\right): v(\mathrm{CO})=2084 \mathrm{vw}, 1928 \mathrm{vs}, 1905 \mathrm{~m}$; $v(\mathrm{CCC})=2011 \mathrm{~m} .{ }^{1} \mathrm{H}-\mathrm{NMR}\left(400 \mathrm{MHz}, \mathrm{CDCl}_{3}\right): \delta=$ $1.46\left(\mathrm{t},{ }^{3} \mathrm{~J}_{\mathrm{HH}}=7.1 \mathrm{~Hz}, 3 \mathrm{H}, \mathrm{OCH}_{2} \mathrm{CH}_{3}\right) 3.14(\mathrm{~s}, 3 \mathrm{H}$, $\left.\mathrm{NCH}_{3}\right), 3.46\left(\mathrm{~s}, 3 \mathrm{H}, \mathrm{NCH}_{3}\right), 4.67\left(\mathrm{q},{ }^{3} J_{\mathrm{HH}}=7.1 \mathrm{~Hz}, 3 \mathrm{H}\right.$, $\left.3 \mathrm{H}, \mathrm{OCH}_{2} \mathrm{CH}_{3}\right) .{ }^{13} \mathrm{C}-\mathrm{NMR}\left(100.5 \mathrm{MHz}, \mathrm{CDCl}_{3}\right): \delta=$ $14.5\left(\mathrm{OCH}_{2} \mathrm{CH}_{3}\right), 28.1,36.9\left(\mathrm{NCH}_{3}\right), 70.8\left(\mathrm{OCH}_{2} \mathrm{CH}_{3}\right)$, $100.4\left({ }^{2} J_{\mathrm{WC}}=25.2 \mathrm{~Hz}, \mathrm{C}_{\beta}\right), 149.8\left(\mathrm{C}_{\gamma}\right), 184.3\left({ }^{1} J_{\mathrm{WC}}=\right.$ $\left.100.6 \mathrm{~Hz}, \mathrm{C}_{\alpha}\right), 197.0\left({ }^{1} J_{\mathrm{WC}}=124.7 \mathrm{~Hz}\right.$, cis-CO $), 201.4$ $\left({ }^{1} J_{\mathrm{WC}}=130.0 \mathrm{~Hz}\right.$, trans $\left.-\mathrm{CO}\right) . \mathrm{MS}(\mathrm{EI}), \mathrm{m} / z(\%): 449$
(65) $\left[\mathrm{M}^{+}\right], 421(43)\left[\mathrm{M}^{+}-\mathrm{CO}\right], 393(100)\left[\mathrm{M}^{+}-2 \mathrm{CO}\right]$, 309 (76) $\left[\mathrm{M}^{+}-5 \mathrm{CO}\right], 307$ (44) $\left[\mathrm{M}^{+}-4 \mathrm{CO}-2 \mathrm{Me}\right], 279$

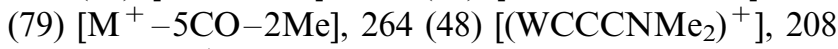
(38) $\left[(\mathrm{WCC})^{+}\right]$. UV-vis: $\lambda_{\max }(\log \varepsilon)$ [solvent]: $428 \mathrm{~nm}$ (4.422) [pentane]; $410 \mathrm{~nm}$ (4.279) $\left[\mathrm{CHCl}_{3}\right] ; 398 \mathrm{~nm}$ (4.218) $\left[\mathrm{CH}_{2} \mathrm{Cl}_{2}\right] ; 376 \mathrm{~nm}$ (4.083) [DMF]. Anal. Found: $\mathrm{C}, 32.18 ; \mathrm{H}, 2.56 ; \mathrm{N}, 3.22$. Calc. for $\mathrm{C}_{12} \mathrm{H}_{11} \mathrm{NO}_{6} \mathrm{~W}$ (449.07): C, 32.10; H, 2.47; N, 3.12\%.

\subsubsection{Pentacarbonyl(3-methoxy-3-pyrrolidino-1,2- propadienylidene) chromium (3a)}

Yellow solid. Yield: $0.86 \mathrm{~g}(52 \%)$. M.p. $121-122{ }^{\circ} \mathrm{C}$. IR (THF, $\left.\mathrm{cm}^{-1}\right): v(\mathrm{CO})=2081 \mathrm{vw}, 1933 \mathrm{vs}, 1911 \mathrm{~m}$; $v(\mathrm{CCC})=2007 \mathrm{~m} .{ }^{1} \mathrm{H}-\mathrm{NMR}\left(400 \mathrm{MHz}, \mathrm{CDCl}_{3}\right): \delta=$ $2.04\left(\mathrm{~m}, 4 \mathrm{H}, \mathrm{CH}_{2} \mathrm{CH}_{2} \mathrm{CH}_{2} \mathrm{CH}_{2}\right), 3.55\left(\mathrm{~s}, 2 \mathrm{H}, \mathrm{NCH}_{2}\right)$, $3.89\left(\mathrm{~m}, 2 \mathrm{H}, \mathrm{NCH}_{2}\right), 4.19\left(\mathrm{~s}, 3 \mathrm{H}, \mathrm{OCH}_{3}\right) .{ }^{13} \mathrm{C}-\mathrm{NMR}$ $\left(100.5 \quad \mathrm{MHz}, \quad \mathrm{CDCl}_{3}\right): \quad \delta=24.4, \quad 25.0$ $\left(\mathrm{CH}_{2} \mathrm{CH}_{2} \mathrm{CH}_{2} \mathrm{CH}_{2}\right), 47.7,51.5\left(\mathrm{NCH}_{2}\right), 59.8\left(\mathrm{OCH}_{3}\right)$, $102.9\left(\mathrm{C}_{\beta}\right), 146.3\left(\mathrm{C}_{\gamma}\right), 207.6\left(\mathrm{C}_{\alpha}\right), 217.7$ (cis- $\left.\mathrm{CO}\right), 221.8$ (trans-CO). Anal. Found: $\mathrm{C}, 47.40 ; \mathrm{H}, 3.53 ; \mathrm{N}, 4.47$. Calc. for $\mathrm{C}_{13} \mathrm{H}_{11} \mathrm{NO}_{6} \mathrm{Cr}$ (329.23): C, 47.43; H, 3.37; N, $4.25 \%$.

\subsubsection{Pentacarbonyl(3-methoxy-3-pyrrolidino-1,2- propadienylidene) tungsten ( $3 \boldsymbol{b})$}

Yellow solid. Yield: 1.37 g (59\%). M.p. $149-150{ }^{\circ} \mathrm{C}$ (dec.). IR (THF, $\mathrm{cm}^{-1}$ ): $v(\mathrm{CO})=2085 \mathrm{vw}, 1928 \mathrm{vs,}$ $1905 \mathrm{~m} ; \quad v(\mathrm{CCC})=2011 \mathrm{~m} .{ }^{1} \mathrm{H}-\mathrm{NMR} \quad(400 \mathrm{MHz}$, $\left.\mathrm{CDCl}_{3}\right): \delta=2.05\left(\mathrm{~m}, 4 \mathrm{H}, \mathrm{CH}_{2} \mathrm{CH}_{2} \mathrm{CH}_{2} \mathrm{CH}_{2}\right), 3.55$ (s, $\left.2 \mathrm{H}, \mathrm{NCH}_{2}\right), 3.88\left(\mathrm{~m}, 2 \mathrm{H}, \mathrm{NCH}_{2}\right), 4.20\left(\mathrm{~s}, 3 \mathrm{H}, \mathrm{OCH}_{3}\right)$. ${ }^{13} \mathrm{C}-\mathrm{NMR} \quad\left(100.5 \mathrm{MHz}, \quad \mathrm{CDCl}_{3}\right): \quad \delta=24.4, \quad 25.0$ $\left(\mathrm{CH}_{2} \mathrm{CH}_{2} \mathrm{CH}_{2} \mathrm{CH}_{2}\right)$, 47.9, $51.6\left(\mathrm{NCH}_{2}\right), 60.0\left(\mathrm{OCH}_{3}\right)$, $101.3\left({ }^{2} J_{\mathrm{WC}}=25.3 \mathrm{~Hz}, \mathrm{C}_{\beta}\right), 148.2\left(\mathrm{C}_{\gamma}\right), 184.2\left({ }^{1} J_{\mathrm{WC}}\right.$ not detected, $\left.\mathrm{C}_{\alpha}\right), 197.0\left({ }^{1} J_{\mathrm{WC}}=124.7 \mathrm{~Hz}\right.$, cis-CO $), 201.4$ $\left({ }^{1} J_{\mathrm{WC}}=131.4 \mathrm{~Hz}\right.$, trans $\left.-\mathrm{CO}\right)$. Anal. Found: C, 33.81; H, 2.52; $\mathrm{N}, 3.17$. Calc. for $\mathrm{C}_{13} \mathrm{H}_{11} \mathrm{NO}_{6} \mathrm{~W}$ (461.08): $\mathrm{C}, 33.86$; $\mathrm{H}, 2.40 ; \mathrm{N}, 3.04 \%$.

\subsubsection{Pentacarbonyl(3-ethoxy-3-pyrrolidino-1,2- propadienylidene) chromium (4a)}

Yellow solid. Yield: 0.88 g (51\%). M.p. $60-61{ }^{\circ} \mathrm{C}$. IR $\left(\mathrm{THF}, \mathrm{cm}^{-1}\right): v(\mathrm{CO})=2080 \mathrm{vw}, 1932 \mathrm{vs}, 1910 \mathrm{~m}$; $v(\mathrm{CCC})=2007 \mathrm{~m} .{ }^{1} \mathrm{H}-\mathrm{NMR}\left(400 \mathrm{MHz}, \mathrm{CDCl}_{3}\right): \delta=$ $1.44\left(\mathrm{t},{ }^{3} J_{\mathrm{HH}}=7.1 \mathrm{~Hz}, 3 \mathrm{H}, \mathrm{OCH}_{2} \mathrm{CH}_{3}\right), 2.04(\mathrm{~m}, 4 \mathrm{H}$, $\left.\mathrm{CH}_{2} \mathrm{CH}_{2} \mathrm{CH}_{2} \mathrm{CH}_{2}\right), 3.55$ (s, $\left.2 \mathrm{H}, \mathrm{NCH}_{2}\right), 3.89$ (m, $2 \mathrm{H}$, $\left.\mathrm{NCH}_{2}\right), 4.65\left(\mathrm{q},{ }^{3} J_{\mathrm{HH}}=7.1 \mathrm{~Hz}, 3 \mathrm{H}, \mathrm{OCH}_{2} \mathrm{CH}_{3}\right) .{ }^{13} \mathrm{C}-$ NMR (100.5 MHz, $\left.\mathrm{CDCl}_{3}\right): \delta=14.6\left(\mathrm{OCH}_{2} \mathrm{CH}_{3}\right), 24.4$, $25.0 \quad\left(\mathrm{CH}_{2} \mathrm{CH}_{2} \mathrm{CH}_{2} \mathrm{CH}_{2}\right), \quad 47.7, \quad 51.3 \quad\left(\mathrm{NCH}_{2}\right), 69.8$ $\left(\mathrm{OCH}_{2} \mathrm{CH}_{3}\right), 102.9\left(\mathrm{C}_{\beta}\right), 145.8\left(\mathrm{C}_{\gamma}\right), 205.4\left(\mathrm{C}_{\alpha}\right), 217.8$ (cis-CO), 221.8 (trans-CO). MS (EI), $\mathrm{m} / \mathrm{z}$ (\%): 303 (14) $\left[\mathrm{M}^{+}\right], 219$ (6) $\left[\mathrm{M}^{+}-3 \mathrm{CO}\right], 191$ (19) $\left[\mathrm{M}^{+}-4 \mathrm{CO}\right], 163$ (100) $\left[\mathrm{M}^{+}-5 \mathrm{CO}\right]$. Anal. Found: C, 48.98; H, 3.91; N, 4.20. Calc. for $\mathrm{C}_{14} \mathrm{H}_{13} \mathrm{NO}_{6} \mathrm{Cr}$ (343.26): C, 48.99; H, 3.82; $\mathrm{N}, 4.08 \%$. 
3.2.8. Pentacarbonyl(3-ethoxy-3-pyrrolidino-1,2-

propadienylidene) tungsten $(\mathbf{4 b})$

Yellow solid. Yield: 1.89 g $(80 \%)$. M.p. $85-86{ }^{\circ}$ C. IR $\left(\mathrm{THF}, \mathrm{cm}^{-1}\right): v(\mathrm{CO})=2084 \mathrm{vw}, 1927 \mathrm{vs}, 1905 \mathrm{~m}$; $v(\mathrm{CCC})=2010 \mathrm{~m} .{ }^{1} \mathrm{H}-\mathrm{NMR}\left(400 \mathrm{MHz}, \mathrm{CDCl}_{3}\right): \delta=$ $1.45\left(\mathrm{t},{ }^{3} J_{\mathrm{HH}}=7.1 \mathrm{~Hz}, 3 \mathrm{H}, \mathrm{OCH}_{2} \mathrm{CH}_{3}\right), 2.04(\mathrm{~m}, 4 \mathrm{H}$, $\left.\mathrm{CH}_{2} \mathrm{CH}_{2} \mathrm{CH}_{2} \mathrm{CH}_{2}\right), 3.55\left(\mathrm{~s}, 2 \mathrm{H}, \mathrm{NCH}_{2}\right), 3.87(\mathrm{~m}, 2 \mathrm{H}$, $\left.\mathrm{NCH}_{2}\right), 4.64\left(\mathrm{q},{ }^{3} J_{\mathrm{HH}}=7.1 \mathrm{~Hz}, 3 \mathrm{H}, \mathrm{OCH}_{2} \mathrm{CH}_{3}\right) \cdot{ }^{13} \mathrm{C}-$ NMR (100.5 MHz, $\left.\mathrm{CDCl}_{3}\right): \delta=14.6\left(\mathrm{OCH}_{2} \mathrm{CH}_{3}\right), 24.4$, $24.9 \quad\left(\mathrm{CH}_{2} \mathrm{CH}_{2} \mathrm{CH}_{2} \mathrm{CH}_{2}\right), \quad 47.8, \quad 51.4 \quad\left(\mathrm{NCH}_{2}\right), \quad 70.1$ $\left(\mathrm{OCH}_{2} \mathrm{CH}_{3}\right), 101.4\left({ }^{2} J_{\mathrm{WC}}\right.$ not detected, $\left.\mathrm{C}_{\beta}\right), 147.7$ $\left(\mathrm{C}_{\gamma}\right), 182.4\left({ }^{1} J_{\mathrm{WC}}\right.$ not detected, $\left.\mathrm{C}_{\alpha}\right), 197.1\left({ }^{1} J_{\mathrm{WC}}=\right.$ $124.7 \mathrm{~Hz}$, cis-CO), $201.5\left({ }^{1} J_{\mathrm{WC}}=130.9 \mathrm{~Hz}\right.$, trans $\left.-\mathrm{CO}\right)$. MS (EI), m/z (\%): $303(14)\left[\mathrm{M}^{+}\right], 219$ (6) [ $\mathrm{M}^{+}-3 \mathrm{CO}$, 191 (19) $\left[\mathrm{M}^{+}{ }_{-} 4 \mathrm{CO}\right], 163$ (100) $\left[\mathrm{M}^{+}-5 \mathrm{CO}\right]$ Anal. Found: $\mathrm{C}, \quad 35.58 ; \mathrm{H}, 2.84 ; \mathrm{N}, 2.89$. Calc. for $\mathrm{C}_{14} \mathrm{H}_{13} \mathrm{NO}_{6} \mathrm{~W}$ (475.11): C, 35.39; H, 2.76; N, 2.95\%.

\subsubsection{Pentacarbonyl(3-diphenylamino-3-methoxy-1,2- propadienylidene) chromium (5a)}

Orange solid. Yield: $0.90 \mathrm{~g}(42 \%)$. M.p. $124-125^{\circ} \mathrm{C}$. IR $\left(\mathrm{THF}, \mathrm{cm}^{-1}\right): v(\mathrm{CO})=2081 \mathrm{vw}, 1937 \mathrm{vs}, 1917 \mathrm{~m}$; $v(\mathrm{CCC})=1996 \mathrm{~m} .{ }^{1} \mathrm{H}-\mathrm{NMR}\left(250 \mathrm{MHz}, \mathrm{CDCl}_{3}\right): \delta=$ $4.25\left(\mathrm{~s}, 3 \mathrm{H}, \mathrm{OCH}_{3}\right), 7.36(\mathrm{~m}, 10 \mathrm{H}, \mathrm{ArH}) .{ }^{13} \mathrm{C}-\mathrm{NMR}$ $\left(62.5 \mathrm{MHz}, \mathrm{CDCl}_{3}\right): \delta=60.6\left(\mathrm{OCH}_{3}\right), 106.9\left(\mathrm{C}_{\beta}\right), 126.2$, $126.9,128.7,128.9,129.2,129.6,139.8,141.8(\mathrm{ArH})$, $144.9\left(\mathrm{C}_{\gamma}\right), 216.9$ (cis-CO), 221.7 (trans $\left.-\mathrm{CO}\right), 225.2\left(\mathrm{C}_{\alpha}\right)$. MS (EI), m/z (\%): $427(10)\left[\mathrm{M}^{+}\right], 343(5)\left[\mathrm{M}^{+}-3 \mathrm{CO}\right]$, 315 (24) $\left[\mathrm{M}^{+}-4 \mathrm{CO}\right], 287$ (100) $\left[\mathrm{M}^{+}-5 \mathrm{CO}\right]$. UV-vis: $\lambda_{\max }(\log \varepsilon)$ [solvent]: $469 \mathrm{~nm}(4.365)$ [pentane]; $450 \mathrm{~nm}$ (4.292) $\left[\mathrm{CHCl}_{3}\right] ; 440 \mathrm{~nm}$ (4.275) $\left[\mathrm{CH}_{2} \mathrm{Cl}_{2}\right] ; 413 \mathrm{~nm}$ (4.215) [DMF]. Anal. Found: C, 59.07; H, 3.19; N, 3.38. Calc. for $\mathrm{C}_{21} \mathrm{H}_{13} \mathrm{NO}_{6} \mathrm{Cr}$ (427.33): C, 59.02; H, 3.07; $\mathrm{N}, 3.28 \%$.

\subsubsection{Pentacarbonyl(3-diphenylamino-3-methoxy-1,2-}

propadienylidene) tungsten $(\mathbf{5} \boldsymbol{b})$

Orange solid. Yield: 1.84 g $(66 \%)$. M.p. $135^{\circ} \mathrm{C}$. IR $\left(\mathrm{THF}, \mathrm{cm}^{-1}\right): v(\mathrm{CO})=2086$ vw, 1933 vs, $1909 \mathrm{~m}$; $v(\mathrm{CCC})=1999 \mathrm{~m} .{ }^{1} \mathrm{H}-\mathrm{NMR}\left(400 \mathrm{MHz}, \mathrm{CDCl}_{3}\right): \delta=$ $4.23\left(\mathrm{~s}, 3 \mathrm{H}, \mathrm{OCH}_{3}\right), 7.23\left(\mathrm{~d},{ }^{3} \mathrm{~J}_{\mathrm{HH}}=7.4 \mathrm{~Hz}, 2 \mathrm{H}, \mathrm{ArH}\right)$, 7.43-7.64 (m, 8H, ArH). ${ }^{13} \mathrm{C}-\mathrm{NMR}(100.5 \mathrm{MHz}$, $\left.\mathrm{CDCl}_{3}\right): \delta=60.8\left(\mathrm{OCH}_{3}\right), 104.9\left({ }^{2} J_{\mathrm{WC}}=25.9 \mathrm{~Hz}, \mathrm{C}_{\beta}\right)$, $125.9,126.7,128.8,129.0,129.6,129.9,139.4,141.5$ $(\mathrm{ArC}), 147.7\left(\mathrm{C}_{\gamma}\right), 196.4\left({ }^{1} J_{\mathrm{WC}}=125.0 \mathrm{~Hz}\right.$, cis-CO $)$, $199.5\left({ }^{1} J_{\mathrm{WC}}=104.8 \mathrm{~Hz}, \mathrm{C}_{\alpha}\right), 201.7\left({ }^{1} J_{\mathrm{WC}}=127.9 \mathrm{~Hz}\right.$, trans-CO). MS (EI), m/z (\%): $559(38)\left[\mathrm{M}^{+}\right], 531(28)$ [M $\left.{ }^{+}-\mathrm{CO}\right], 475(48)\left[\mathrm{M}^{+}-3 \mathrm{CO}\right], 449$ (37) $\left[\mathrm{M}^{+}-4 \mathrm{CO}\right]$, $419(100)\left[\mathrm{M}^{+}-5 \mathrm{CO}\right], 169(35)\left[\left(\mathrm{C}_{12} \mathrm{H}_{11} \mathrm{~N}\right)^{+}\right]$. UV-vis: $\lambda_{\max }(\log \varepsilon)$ [solvent]: $462 \mathrm{~nm}(4.466)$ [pentane]; $444 \mathrm{~nm}$ (4.275) $\left[\mathrm{CHCl}_{3}\right] ; 435 \mathrm{~nm}$ (4.366) $\left[\mathrm{CH}_{2} \mathrm{Cl}_{2}\right] ; 412 \mathrm{~nm}$ (4.264) [DMF]. Anal. Found: C, 45.15; H, 2.41; N, 2.63. Calc. for $\mathrm{C}_{21} \mathrm{H}_{13} \mathrm{NO}_{6} \mathrm{~W}$ (559.19): $\mathrm{C}, 45.11 ; \mathrm{H}, 2.34$; $\mathrm{N}, 2.50 \%$.
3.2.11. Pentacarbonyl(3-diphenylamino-3-ethoxy-1,2propadienylidene) chromium (6a)

Orange solid. Yield: $1.04 \mathrm{~g}(47 \%)$. M.p. $107-108{ }^{\circ} \mathrm{C}$. IR $\left(\mathrm{THF}, \mathrm{cm}^{-1}\right): v(\mathrm{CO})=2080 \mathrm{vw}, 1936 \mathrm{vs}, 1918 \mathrm{~m}$; $v(\mathrm{CCC})=1997 \mathrm{~m} .{ }^{1} \mathrm{H}-\mathrm{NMR}\left(250 \mathrm{MHz}, \mathrm{CDCl}_{3}\right): \delta=$ $1.41\left(\mathrm{t},{ }^{3} J_{\mathrm{HH}}=7.1 \mathrm{~Hz}, 3 \mathrm{H}, \mathrm{OCH}_{2} \mathrm{CH}_{3}\right), 4.73\left(\mathrm{q},{ }^{3} J_{\mathrm{HH}}=\right.$ $\left.7.1 \mathrm{~Hz}, 2 \mathrm{H}, \mathrm{OCH}_{2} \mathrm{CH}_{3}\right), 7.37(\mathrm{~m}, 10 \mathrm{H}, \mathrm{ArH}) .{ }^{13} \mathrm{C}-\mathrm{NMR}$ $\left(62.5 \mathrm{MHz}, \quad \mathrm{CDCl}_{3}\right): \quad \delta=14.3 \quad\left(\mathrm{OCH}_{2} \mathrm{CH}_{3}\right), \quad 70.9$ $\left(\mathrm{OCH}_{2} \mathrm{CH}_{3}\right), 106.6\left(\mathrm{C}_{\beta}\right), 126.2,127.0,127.9,128.5$, 128.8, 129.6, 139.9, 141.8 (ArC), $144.5\left(\mathrm{C}_{\gamma}\right), 217.0$ (cisCO), 221.8 (trans-CO), $222.4\left(\mathrm{C}_{\alpha}\right)$. MS (EI), $\mathrm{m} / z(\%)$ : $441(17)\left[\mathrm{M}^{+}\right], 329$ (29) $\left[\mathrm{M}^{+}{ }_{-} 4 \mathrm{CO}\right], 301(100)\left[\mathrm{M}^{+}{ }_{-}\right.$ 5CO]. UV-vis: $\lambda_{\max }(\log \varepsilon)$ [solvent]: $467 \mathrm{~nm}(4.383)$ [pentane]; $447 \mathrm{~nm}$ (4.314) $\left[\mathrm{CHCl}_{3}\right] ; 438 \mathrm{~nm}$ (4.306) $\left[\mathrm{CH}_{2} \mathrm{Cl}_{2}\right] ; 413 \mathrm{~nm}$ (4.224) [DMF]. Anal. Found: C, 59.82; $\mathrm{H}, 3.55 ; \mathrm{N}, 3.30$. Calc. for $\mathrm{C}_{22} \mathrm{H}_{15} \mathrm{NO}_{6} \mathrm{Cr}$ (441.36): C, 59.87; H, 3.43; N, 3.17\%.

\subsubsection{Pentacarbonyl(3-diphenylamino-3-ethoxy-1,2- propadienylidene) tungsten (6b)}

Orange solid. Yield: 2.01 g $(70 \%)$. M.p. $121-123{ }^{\circ} \mathrm{C}$. IR $\left(\mathrm{THF}, \mathrm{cm}^{-1}\right): v(\mathrm{CO})=2086 \mathrm{vw}, 1932 \mathrm{vs}, 1909 \mathrm{~m}$; $v(\mathrm{CCC})=1999 \mathrm{~m} .{ }^{1} \mathrm{H}-\mathrm{NMR}\left(400 \mathrm{MHz}, \mathrm{CDCl}_{3}\right): \delta=$ $1.33\left(\mathrm{t},{ }^{3} J_{\mathrm{HH}}=7.1 \mathrm{~Hz}, 3 \mathrm{H}, \mathrm{OCH}_{2} \mathrm{CH}_{3}\right), 4.64\left(\mathrm{q},{ }^{3} J_{\mathrm{HH}}=\right.$ $\left.7.1 \mathrm{~Hz}, 2 \mathrm{H}, \mathrm{OCH}_{2} \mathrm{CH}_{3}\right), 7.14\left(\mathrm{~d},{ }^{3} J_{\mathrm{HH}}=5.5 \mathrm{~Hz}, 2 \mathrm{H}\right.$, ArH), 7.26-7.39 (m, 8H, ArH). ${ }^{13} \mathrm{C}-\mathrm{NMR}(100.5 \mathrm{MHz}$, $\left.\mathrm{CDCl}_{3}\right): \delta=14.2\left(\mathrm{OCH}_{2} \mathrm{CH}_{3}\right), 71.2\left(\mathrm{OCH}_{2} \mathrm{CH}_{3}\right), 104.6$ $\left({ }^{2} J_{\mathrm{WC}}=26.0 \mathrm{~Hz}, \mathrm{C}_{\beta}\right), 125.9,126.8,128.3,128.6,128.9$, $129.5,139.5,141.5(\mathrm{ArC}), 147.2\left(\mathrm{C}_{\gamma}\right), 196.4\left({ }^{1} J_{\mathrm{WC}}=\right.$ $125.0 \mathrm{~Hz}$, cis-CO), $197.2\left({ }^{1} J_{\mathrm{WC}}=101.2 \mathrm{~Hz}, \mathrm{C}_{\alpha}\right), 201.7$ $\left({ }^{1} J_{\mathrm{WC}}=126.9 \mathrm{~Hz}\right.$, trans-CO). MS (EI), $\mathrm{m} / \mathrm{z}(\%): 573$ (53) $\left[\mathrm{M}^{+}\right], 545(36)\left[\mathrm{M}^{+}-\mathrm{CO}\right], 487$ (18) $\left[\mathrm{M}^{+}-3 \mathrm{CO}\right]$, 461 (100) $\left[\mathrm{M}^{+}-4 \mathrm{CO}\right], 433$ (42) $\left[\mathrm{M}^{+}-5 \mathrm{CO}\right], 169$ (51) $\left[\left(\mathrm{C}_{12} \mathrm{H}_{11} \mathrm{~N}\right)^{+}\right]$. UV-vis: $\lambda_{\max }(\log \varepsilon)$ [solvent]: $460 \mathrm{~nm}$ (4.500) [pentane]; $442 \mathrm{~nm}$ (4.390) $\left[\mathrm{CHCl}_{3}\right] ; 433 \mathrm{~nm}$ (4.353) $\left[\mathrm{CH}_{2} \mathrm{Cl}_{2}\right] ; 412 \mathrm{~nm}$ (4.252) [DMF]. Anal. Found: $\mathrm{C}, 45.84 ; \mathrm{H}, 2.61 ; \mathrm{N}, 2.53$. Calc. for $\mathrm{C}_{22} \mathrm{H}_{15} \mathrm{NO}_{6} \mathrm{~W}$ (573.21): C, 46.10; H, 2.64; N, 2.44\%.

\subsubsection{Pentacarbonyl (3-dimethylamino-3-phenyl-1,2-} propadienylidene) chromium ( 7 a)

Yield: $1.06 \mathrm{~g}(61 \%)$. Identification through comparison of its spectroscopic data with those published in the literature $[13,15,16 \mathrm{c}]$.

\subsubsection{Pentacarbonyl(3-dimethylamino-3-phenyl-1,2- propadienylidene) tungsten ( $7 \boldsymbol{b})$}

Yield: $1.66 \mathrm{~g}(69 \%)$. Identification through comparison of its spectroscopic data with those published in the literature $[13,15,16 \mathrm{c}]$.

\subsubsection{Pentacarbonyl(3-ethylmethylamino-3-phenyl-1,2-} propadienylidene) chromium (8a)

Maroon solid. Yield: $1.13 \mathrm{~g} \mathrm{(62 \% ).} \mathrm{M.p.} 84-85^{\circ} \mathrm{C}$. IR $\left(\mathrm{THF}, \mathrm{cm}^{-1}\right): v(\mathrm{CO})=2075 \mathrm{vw}, 1935 \mathrm{vs}, 1912 \mathrm{~m}$; $v(\mathrm{CCC})=1994 \mathrm{~m} .{ }^{1} \mathrm{H}-\mathrm{NMR}\left(400 \mathrm{MHz}, \mathrm{CDCl}_{3}\right): \delta=$ 
$1.28\left(\mathrm{t},{ }^{3} J_{\mathrm{HH}}=7.4 \mathrm{~Hz}, 3 \mathrm{H}, \mathrm{NEt}\right), 1.48\left(\mathrm{t},{ }^{3} J_{\mathrm{HH}}=7.4 \mathrm{~Hz}\right.$, $3 \mathrm{H}, \mathrm{NEt}), 3.25(\mathrm{~s}, 3 \mathrm{H}, \mathrm{NMe}), 3.59\left(\mathrm{q},{ }^{3} J_{\mathrm{HH}}=7.4 \mathrm{~Hz}\right.$, $2 \mathrm{H}, \mathrm{NEt}), 3.64$ (s, 3H, NMe), 4.09 (q, ${ }^{3} J_{\mathrm{HH}}=7.4 \mathrm{~Hz}$, 2H, NEt), $7.60(\mathrm{~m}, 5 \mathrm{H}, \mathrm{ArH}) .{ }^{13} \mathrm{C}-\mathrm{NMR}(100.5 \mathrm{MHz}$, $\left.\mathrm{CDCl}_{3}\right): \delta=11.4,13.3(\mathrm{NEt}), 40.0,41.3(\mathrm{NMe}), 49.4$, $52.4(\mathrm{NEt}), 123.2,124.7\left(\mathrm{C}_{\beta}\right), 127.7,128.4,128.5,128.6$, $128.8,131.0,131.4,134.2,134,4$ (ArC), 152.8, 153.6 $\left(\mathrm{C}_{\gamma}\right), 217.4$ (cis-CO), 223.9, 224.0 (trans-CO), 226.4, $229.7\left(\mathrm{C}_{\alpha}\right)$. MS (EI), $\mathrm{m} / \mathrm{z}(\%): 363(4)\left[\mathrm{M}^{+}\right], 307$ (1) [M $\left.\mathrm{M}^{+}-2 \mathrm{CO}\right], 279$ (2) $\left[\mathrm{M}^{+}-3 \mathrm{CO}\right], 251$ (8) $\left[\mathrm{M}^{+}{ }_{-} 4 \mathrm{CO}\right]$, 223 (100) $\left[\mathrm{M}^{+}-5 \mathrm{CO}\right.$. UV-vis: $\lambda_{\max }(\log \varepsilon)$ [solvent]: $522 \mathrm{~nm}$ (4.306) [pentane]; $490 \mathrm{~nm}$ (4.282) $\left[\mathrm{CHCl}_{3}\right] ; 477$ $\mathrm{nm}$ (4.273) $\left[\mathrm{CH}_{2} \mathrm{Cl}_{2}\right] ; 443 \mathrm{~nm}$ (4.205) [DMF]. Anal. Found: C, 56.23; H, 3.67; N, 3.90. Calc. for $\mathrm{C}_{17} \mathrm{H}_{13} \mathrm{NO}_{5} \mathrm{Cr}$ (363.29): C, 56.20; H, 3.61; N, 3.86\%.

\subsubsection{Pentacarbonyl(3-ethylmethylamino-3-phenyl-1,2- propadienylidene) tungsten $(\boldsymbol{8} \boldsymbol{b})$}

Maroon solid. Yield: $1.68 \mathrm{~g}(68 \%)$. M.p. $113-115^{\circ} \mathrm{C}$. IR $\left(\mathrm{THF}, \mathrm{cm}^{-1}\right): v(\mathrm{CO})=2080 \mathrm{vw}, 1929 \mathrm{vs}, 1905 \mathrm{~m}$; $v(\mathrm{CCC})=1995 \mathrm{~m} .{ }^{1} \mathrm{H}-\mathrm{NMR}\left(400 \mathrm{MHz}, \mathrm{CDCl}_{3}\right): \delta=$ $1.24\left(\mathrm{t},{ }^{3} J_{\mathrm{HH}}=7.4 \mathrm{~Hz}, 3 \mathrm{H}, \mathrm{NEt}\right), 1.44\left(\mathrm{t},{ }^{3} J_{\mathrm{HH}}=7.4 \mathrm{~Hz}\right.$, $3 \mathrm{H}, \mathrm{NEt}$ ), 3.18 (s, $3 \mathrm{H}, \mathrm{NMe}), 3.54$ (q, ${ }^{3} J_{\mathrm{HH}}=7.4 \mathrm{~Hz}$, $2 \mathrm{H}, \mathrm{NEt}), 3.56(\mathrm{~s}, 3 \mathrm{H}, \mathrm{NMe}), 4.02\left(\mathrm{q},{ }^{3} J_{\mathrm{HH}}=7.4 \mathrm{~Hz}\right.$, 2H, NEt), 7.42 (m, 5H, ArH). ${ }^{13} \mathrm{C}-\mathrm{NMR}(100.5 \mathrm{MHz}$, $\left.\mathrm{CDCl}_{3}\right): \delta=11.6,13.3(\mathrm{NEt}), 40.3,41.6(\mathrm{NMe}), 49.7$, $52.7(\mathrm{NEt}), 121.5,123.1\left(\mathrm{C}_{\beta},{ }^{2} J_{\mathrm{WC}}=25.0 \mathrm{~Hz}\right), 127.6$, $128.4,128.6,128.8,128.9,131.2,131.5,134.2,134.4$ (ArC), 154.7, $155.4\left(\mathrm{C}_{\gamma}\right), 196.9\left({ }^{1} J_{\mathrm{WC}}=125.9 \mathrm{~Hz}\right.$, cis CO), $203.9\left({ }^{1} J_{\mathrm{WC}}=127.4 \mathrm{~Hz}\right.$, trans-CO $), 204.6,207.5$ $\left({ }^{1} J_{\mathrm{WC}}=\right.$ not detected, $\left.\mathrm{C}_{\alpha}\right) . \mathrm{MS}(\mathrm{EI}), \mathrm{m} / \mathrm{z}(\%): 495$ (2) $\left[\mathrm{M}^{+}\right], 467$ (2) $\left[\mathrm{M}^{+}-\mathrm{CO}\right], 439$ (3) $\left[\mathrm{M}^{+}-2 \mathrm{CO}\right], 385$ (4) $\left[\mathrm{M}^{+}-4 \mathrm{CO}\right], 352(30)\left[\mathrm{W}(\mathrm{CO})_{6}^{+}\right], 296(63)\left[\mathrm{W}(\mathrm{CO})_{4}^{+}\right]$, $268(85)\left[\mathrm{W}(\mathrm{CO})_{3}^{+}\right], 184(100)\left[\mathrm{W}^{+}\right]$. UV-vis: $\lambda_{\max }$ $(\log \varepsilon)$ [solvent]: $506 \mathrm{~nm}$ (4.443) [pentane]; $478 \mathrm{~nm}$ (4.371) $\left[\mathrm{CHCl}_{3}\right] ; 465 \mathrm{~nm}$ (4.358) $\left[\mathrm{CH}_{2} \mathrm{Cl}_{2}\right] ; 434 \mathrm{~nm}$ (4.273) [DMF]. Anal. Found: C, 41.54; H, 2.77; N, 3.04. Calc. for $\mathrm{C}_{17} \mathrm{H}_{13} \mathrm{NO}_{5} \mathrm{~W}$ (303.19): C, 41.24; H, 2.65; $\mathrm{N}, 2.83 \%$.

\subsubsection{Pentacarbonyl(3-methylamino-3-phenyl-1,2- propadienylidene) chromium $(\mathbf{9 a})$}

Maroon solid. Yield: 1.31 g (78\%). M.p. $121-123{ }^{\circ} \mathrm{C}$ (dec.). IR (THF, $\left.\mathrm{cm}^{-1}\right): v(\mathrm{CO})=2077 \mathrm{vw}, 1936 \mathrm{vs,}$ $1914 \mathrm{~m} ; v(\mathrm{CCC})=1994 \mathrm{~m} .{ }^{1} \mathrm{H}-\mathrm{NMR}\left(400 \mathrm{MHz},\left[d_{6}\right]-\right.$ acetone $): \delta=3.56(\mathrm{~s}, 3 \mathrm{H}, \mathrm{NMe}), 7.56\left(\mathrm{t},{ }^{3} J_{\mathrm{HH}}=7.4 \mathrm{~Hz}\right.$, $2 \mathrm{H}, \mathrm{ArH}), 7.66\left(\mathrm{t},{ }^{3} J_{\mathrm{HH}}=7.4 \mathrm{~Hz}, 1 \mathrm{H}, \operatorname{ArH}\right), 8.09(\mathrm{~d}$, $\left.{ }^{3} J_{\mathrm{HH}}=7.4 \mathrm{~Hz}, 2 \mathrm{H}, \mathrm{ArH}\right), 10.54(\mathrm{br}, 1 \mathrm{H}, \mathrm{NH}),{ }^{13} \mathrm{C}-$ NMR (100.5 MHz, $\left[d_{6}\right]$-acetone): $\delta=35.1(\mathrm{NMe}), 118.4$ $\left(\mathrm{C}_{\beta}\right), 129.6,129.9,133.2,134.5(\mathrm{ArC}), 153.4\left(\mathrm{C}_{\gamma}\right), 218.8$ (cis-CO), $220.3\left(\mathrm{C}_{\alpha}\right), 224.3$ (trans-CO). MS (EI), $\mathrm{m} / \mathrm{z}$ (\%): $335(10)\left[\mathrm{M}^{+}\right], 251(8)\left[\mathrm{M}^{+}-3 \mathrm{CO}\right], 195(80)\left[\mathrm{M}^{+}{ }_{-}\right.$ 5CO], 108 (100) $\left[\left(\mathrm{Cr}(\mathrm{CO})_{2}^{+}\right] . \quad \mathrm{UV}\right.$-vis: $\lambda_{\max }(\log \varepsilon)$ [solvent]: $564 \mathrm{~nm}$ (3.841) [pentane]; $519 \mathrm{~nm}$ (4.248) $\left[\mathrm{CHCl}_{3}\right] ; 514 \mathrm{~nm}(4.252)\left[\mathrm{CH}_{2} \mathrm{Cl}_{2}\right] ; 462 \mathrm{~nm}$ (3.814)
[DMF]. Anal. Found: C, 53.65; H, 2.73; N, 4.22. Calc. for $\mathrm{C}_{15} \mathrm{H}_{9} \mathrm{NO}_{5} \mathrm{Cr}$ (335.24): C, 53.74; H, 2.71; N, 2.71\%.

\subsubsection{Pentacarbonyl(3-methylamino-3-phenyl-1,2- propadienylidene) tungsten ( $9 \boldsymbol{b})$}

Maroon solid. Yield: $1.77 \mathrm{~g}(76 \%)$. M.p. $145^{\circ} \mathrm{C}$ (dec.). IR $\left(\right.$ THF, $\left.\mathrm{cm}^{-1}\right): v(\mathrm{CO})=2082 \mathrm{vw}, 1930 \mathrm{vs}, 1906 \mathrm{~m}$; $v(\mathrm{CCC})=1996 \mathrm{~m} .{ }^{1} \mathrm{H}-\mathrm{NMR}\left(400 \mathrm{MHz},\left[d_{6}\right]\right.$-acetone $)$ : $\delta=3.56(\mathrm{~s}, 3 \mathrm{H}, \mathrm{NMe}), 7.57\left(\mathrm{t},{ }^{3} J_{\mathrm{HH}}=7.4 \mathrm{~Hz}, 2 \mathrm{H}\right.$, $\operatorname{ArH}), 7.71\left(\mathrm{t},{ }^{3} J_{\mathrm{HH}}=7.4 \mathrm{~Hz}, 1 \mathrm{H}, \operatorname{ArH}\right), 8.08\left(\mathrm{~d},{ }^{3} J_{\mathrm{HH}}=\right.$ $7.4 \mathrm{~Hz}, 2 \mathrm{H}, \mathrm{ArH}), 10.70$ (br, 1H, NH). ${ }^{13} \mathrm{C}-\mathrm{NMR}(100.5$ $\mathrm{MHz},\left[d_{6}\right]$-acetone $): \delta=35.6(\mathrm{NMe}), 115.9\left({ }^{2} J_{\mathrm{WC}}=25.0\right.$ $\left.\mathrm{Hz}, \mathrm{C}_{\beta}\right), 129.6,130.0,133.2,134.6(\mathrm{ArC}), 155.5\left(\mathrm{C}_{\gamma}\right)$, $196.4\left({ }^{1} J_{\mathrm{WC}}=102.0 \mathrm{~Hz}, \mathrm{C}_{\alpha}\right), 198.0\left({ }^{1} J_{\mathrm{WC}}=123.2 \mathrm{~Hz}\right.$, cis-CO), $203.6\left({ }^{1} J_{\mathrm{WC}}=129.0 \mathrm{~Hz}\right.$, trans-CO). MS (EI), $\mathrm{m} / \mathrm{z}$ (\%): 467 (10) $\left[\mathrm{M}^{+}\right], 439$ (11) $\left[\mathrm{M}^{+}-\mathrm{CO}\right], 411$ (13) [M+ $\left.\mathrm{M}^{+}-3 \mathrm{CO}\right], 383$ (9) $\left[\mathrm{M}^{+}-4 \mathrm{CO}\right], 355$ (6) $\left[\mathrm{M}^{+}-5 \mathrm{CO}\right]$, $184(100)\left[\mathrm{W}^{+}\right.$]. UV-vis: $\lambda_{\max }(\log \varepsilon)$ [solvent]: $549 \mathrm{~nm}$ (3.551) [pentane]; $502 \mathrm{~nm}$ (4.280) $\left[\mathrm{CHCl}_{3}\right] ; 496 \mathrm{~nm}$ (4.335) $\left[\mathrm{CH}_{2} \mathrm{Cl}_{2}\right] ; 449 \mathrm{~nm}$ (3.913) [DMF]. Anal. Found: C, 38.72; H, 2.12; N, 3.16. Calc. for $\mathrm{C}_{15} \mathrm{H}_{9} \mathrm{NO}_{5} \mathrm{~W}$ (467.09): C, 38.57; H, 1.94; N, 3.00\%.

\subsubsection{Pentacarbonyl(3-hydro- $N$-methylpyridine-1,2- propadienylidene) chromium (10a)}

Orange solid. Yield: 1.16 g (75\%). M.p. $170{ }^{\circ} \mathrm{C}$ (dec.). IR (THF, $\left.\mathrm{cm}^{-1}\right): v(\mathrm{CO})=2076 \mathrm{vw}, 1929$ vs, $1901 \mathrm{~m}$; $v(\mathrm{CCC})=2012 \mathrm{~m} .{ }^{1} \mathrm{H}-\mathrm{NMR}\left(400 \mathrm{MHz},\left[d_{6}\right]\right.$-acetone $)$ : $\delta=4.31\left(\mathrm{~s}, 3 \mathrm{H}, \mathrm{NCH}_{3}\right), 7.53\left(\mathrm{t},{ }^{3} J_{\mathrm{HH}}=7.0 \mathrm{~Hz}, 1 \mathrm{H}\right.$, PyrH), $7.61\left(\mathrm{~d},{ }^{3} J_{\mathrm{HH}}=7.0 \mathrm{~Hz}, 1 \mathrm{H}, \operatorname{PyrH}\right), 8.17(\mathrm{t}$, $\left.{ }^{3} J_{\mathrm{HH}}=7.0 \mathrm{~Hz}, 1 \mathrm{H}, \mathrm{PyrH}\right), 8.60\left(\mathrm{~d},{ }^{3} J_{\mathrm{HH}}=7.0 \mathrm{~Hz}, 1 \mathrm{H}\right.$, PyrH). ${ }^{13} \mathrm{C}-\mathrm{NMR}\left(100.5 \mathrm{MHz},\left[d_{6}\right]\right.$-acetone): $\delta=46.6$ $\left(\mathrm{NCH}_{3}\right), 111.6\left(\mathrm{C}_{\beta}\right), 120.8,130.2,143.1,144.7$ (4 PyrC), $139.0\left(\mathrm{C}_{\gamma}\right), 186.1\left(\mathrm{C}_{\alpha}\right), 219.6$ (cis-CO), 223.7 (trans $\left.-\mathrm{CO}\right)$. MS (EI), $m / z(\%): 309(25)\left[\mathrm{M}^{+}\right], 281(16)\left[\mathrm{M}^{+}-\mathrm{CO}\right]$, 253 (21) $\left[\mathrm{M}^{+}{ }_{-} \mathrm{CCO}\right], 197(22)\left[\mathrm{M}^{+}{ }_{-4} \mathrm{CO}\right], 169$ (100) [M ${ }^{+}-5 \mathrm{CO}$. UV-vis: $\lambda_{\max }(\log \varepsilon)$ [solvent]: $502 \mathrm{~nm}$ (4.235) $\left[\mathrm{CHCl}_{3}\right] ; 479 \mathrm{~nm}$ (4.228) $\left[\mathrm{CH}_{2} \mathrm{Cl}_{2}\right] ; 431 \mathrm{~nm}$ (4.217) [DMF]. Anal. Found: C, 50.34; H, 2.51; N, 4.64. Calc. for $\mathrm{C}_{13} \mathrm{H}_{7} \mathrm{NO}_{5} \mathrm{Cr}$ (309.20): C, 50.50; H, 2.28; $\mathrm{N}, 4.53 \%$.

\subsubsection{Pentacarbonyl(3-hydro- $N$-methylpyridine-1,2- propadienylidene) tungsten $(\mathbf{1 0 b}$}

Orange solid. Yield: $1.41 \mathrm{~g}(64 \%)$. M.p. $193{ }^{\circ} \mathrm{C}$ (dec.). IR $\left(\mathrm{THF}, \mathrm{cm}^{-1}\right): v(\mathrm{CO})=2079 \mathrm{vw}, 1922 \mathrm{vs}, 1894 \mathrm{~m}$; $v(\mathrm{CCC})=2016 \mathrm{~m} .{ }^{1} \mathrm{H}-\mathrm{NMR}\left(400 \mathrm{MHz},\left[d_{6}\right]\right.$-acetone): $\delta=4.31\left(\mathrm{~s}, 3 \mathrm{H}, \mathrm{NCH}_{3}\right), 7.57\left(\mathrm{t},{ }^{3} J_{\mathrm{HH}}=7.0 \mathrm{~Hz}, 1 \mathrm{H}\right.$, PyrH), $7.66\left(\mathrm{~d},{ }^{3} J_{\mathrm{HH}}=7.0 \mathrm{~Hz}, 1 \mathrm{H}, \operatorname{PyrH}\right), 8.20(\mathrm{t}$, $\left.{ }^{3} J_{\mathrm{HH}}=7.0 \mathrm{~Hz}, 1 \mathrm{H}, \mathrm{PyrH}\right), 8.64\left(\mathrm{~d},{ }^{3} J_{\mathrm{HH}}=7.0 \mathrm{~Hz}, 1 \mathrm{H}\right.$, PyrH). ${ }^{13} \mathrm{C}$-NMR (100.5 MHz, $\left[d_{6}\right]$-acetone): $\delta=46.7$ $\left(\mathrm{NCH}_{3}\right), 109.7\left({ }^{2} J_{\mathrm{WC}}=23.0 \mathrm{~Hz}, \mathrm{C}_{\beta}\right), 121.3,130.5,143.2$, 144.9 (4 PyrC), $139.2\left(\mathrm{C}_{\gamma}\right), 166.0\left({ }^{1} J_{\mathrm{WC}}=100.1 \mathrm{~Hz}, \mathrm{C}_{\alpha}\right)$, $198.7\left({ }^{1} J_{\mathrm{WC}}=123.2 \mathrm{~Hz}\right.$, cis $\left.-\mathrm{CO}\right), 202.9\left({ }^{1} J_{\mathrm{WC}}=123.8\right.$ $\mathrm{Hz}$, trans $-\mathrm{CO})$. MS (EI), m/z (\%): $441(23)\left[\mathrm{M}^{+}\right], 385$ (38) $\left[\mathrm{M}^{+}{ }_{-} 2 \mathrm{CO}\right], 329$ (34) [M+ $\left.{ }^{+} 4 \mathrm{CO}\right], 301$ (100) $\left[\mathrm{M}^{+}{ }_{-}\right.$ 
5CO], $272(80)\left[\mathrm{M}^{+}-5 \mathrm{CO}-\mathrm{Et}\right] . \mathrm{UV}-\mathrm{vis}: \lambda_{\max }(\log \varepsilon)$ [solvent]: $485 \mathrm{~nm}$ (4.285) $\left[\mathrm{CHCl}_{3}\right.$ ]; $464 \mathrm{~nm}$ (4.308) $\left[\mathrm{CH}_{2} \mathrm{Cl}_{2}\right] ; 423 \mathrm{~nm}$ (4.319) [DMF]. Anal. Found: C, 35.16; $\mathrm{H}, 1.60 ; \mathrm{N}, 3.21$. Calc. for $\mathrm{C}_{13} \mathrm{H}_{7} \mathrm{NO}_{5} \mathrm{~W}$ (441.05): C, 35.40; H, $1.60 ; \mathrm{N}, 3.18 \%$.

\subsubsection{Pentacarbonyl(N-ethyl-3-hydropyridine-1,2- propadienylidene) chromium (11a)}

Orange solid. Yield: $1.15 \mathrm{~g}(71 \%)$. M.p. $116-118^{\circ} \mathrm{C}$. IR (THF, $\left.\mathrm{cm}^{-1}\right): v(\mathrm{CO})=2076 \mathrm{vw}, 1928 \mathrm{vs}, 1900 \mathrm{~m}$; $v(\mathrm{CCC})=2012 \mathrm{~m} .{ }^{1} \mathrm{H}-\mathrm{NMR}\left(400 \mathrm{MHz},\left[d_{6}\right]\right.$-acetone $)$ $\delta=1.61\left(\mathrm{t},{ }^{3} J_{\mathrm{HH}}=7.1 \mathrm{~Hz}, 3 \mathrm{H}, \mathrm{NCH}_{2} \mathrm{CH}_{3}\right), 4.76(\mathrm{q}$, $\left.{ }^{3} J_{\mathrm{HH}}=7.1 \mathrm{~Hz}, 4 \mathrm{H}, \mathrm{NCH}_{2} \mathrm{CH}_{3}\right), 7.55\left(\mathrm{t},{ }^{3} J_{\mathrm{HH}}=7.0 \mathrm{~Hz}\right.$, $1 \mathrm{H}$, py-H), $7.62\left(\mathrm{~d},{ }^{3} J_{\mathrm{HH}}=7.0 \mathrm{~Hz}, 1 \mathrm{H}\right.$, py $\left.-\mathrm{H}\right), 8.17(\mathrm{t}$, $\left.{ }^{3} J_{\mathrm{HH}}=7.0 \mathrm{~Hz}, 1 \mathrm{H}, \mathrm{PyrH}\right), 8.62\left(\mathrm{~d},{ }^{3} J_{\mathrm{HH}}=7.0 \mathrm{~Hz}, 1 \mathrm{H}\right.$, py-H). ${ }^{13} \mathrm{C}-\mathrm{NMR}\left(100.5 \mathrm{MHz},\left[d_{6}\right]\right.$-acetone): $\delta=15.0$ $\left(\mathrm{NCH}_{2} \mathrm{CH}_{3}\right), \quad 55.0\left(\mathrm{NCH}_{2} \mathrm{CH}_{3}\right), 110.7 \quad\left(\mathrm{C}_{\beta}\right), 121.4$, $130.7,143.2,143.6$ (py-C), $137.9\left(\mathrm{C}_{\gamma}\right), 184.6\left(\mathrm{C}_{\alpha}\right)$, 219.6 (cis-CO), 223.6 (trans-CO). MS (EI), $\mathrm{m} / z(\%)$ : $323(5)\left[\mathrm{M}^{+}\right], 211(6)\left[\mathrm{M}^{+}{ }_{-4 \mathrm{CO}}\right], 183(100)\left[\mathrm{M}^{+}{ }_{-}\right.$ 5CO], $154(20)\left[\mathrm{M}^{+}-5 \mathrm{CO}-\mathrm{Et}\right] . \mathrm{UV}-\mathrm{vis}: \lambda_{\max }(\log \varepsilon)$ [solvent]: $502 \mathrm{~nm}$ (4.226) $\left[\mathrm{CHCl}_{3}\right] ; 479 \mathrm{~nm}$ (4.222) $\left[\mathrm{CH}_{2} \mathrm{Cl}_{2}\right] ; 434 \mathrm{~nm}$ (4.209) [DMF]. Anal. Found: C, 51.73; H, 2.79; N, 4.38. Calc. for $\mathrm{C}_{14} \mathrm{H}_{9} \mathrm{NO}_{5} \mathrm{Cr}(323.26)$ : C, $52.02 ; \mathrm{H}, 2.81 ; \mathrm{N}, 4.33 \%$.

\subsubsection{Pentacarbonyl(N-ethyl-3-hydropyridine-1,2- propadienylidene) tungsten (11b)}

Orange solid. Yield: $1.91 \mathrm{~g}(84 \%)$. M.p. $125-128^{\circ} \mathrm{C}$. IR $\left(\mathrm{THF}, \mathrm{cm}^{-1}\right): v(\mathrm{CO})=2081 \mathrm{vw}, 1922 \mathrm{vs}, 1896 \mathrm{~m}$; $v(\mathrm{CCC})=2015 \mathrm{~m} .{ }^{1} \mathrm{H}-\mathrm{NMR}\left(400 \mathrm{MHz},\left[d_{6}\right]\right.$-acetone $)$ : $\delta=1.63\left(\mathrm{t},{ }^{3} J_{\mathrm{HH}}=7.1 \mathrm{~Hz}, 3 \mathrm{H}, \mathrm{NCH}_{2} \mathrm{CH}_{3}\right), 4.77$ (q, $\left.{ }^{3} J_{\mathrm{HH}}=7.1 \mathrm{~Hz}, 4 \mathrm{H}, \mathrm{NCH}_{2} \mathrm{CH}_{3}\right), 7.60\left(\mathrm{t},{ }^{3} J_{\mathrm{HH}}=7.0 \mathrm{~Hz}\right.$, $1 \mathrm{H}$, py-H), $7.66\left(\mathrm{~d},{ }^{3} J_{\mathrm{HH}}=7.0 \mathrm{~Hz}, 1 \mathrm{H}\right.$, py $\left.-\mathrm{H}\right), 8.22(\mathrm{t}$, ${ }^{3} J_{\mathrm{HH}}=7.0 \mathrm{~Hz}, 1 \mathrm{H}$, py $\left.-\mathrm{H}\right), 8.67\left(\mathrm{~d},{ }^{3} J_{\mathrm{HH}}=7.0 \mathrm{~Hz}, 1 \mathrm{H}\right.$, py-H). ${ }^{13} \mathrm{C}$-NMR $\left(100.5 \mathrm{MHz},\left[d_{6}\right]\right.$-acetone $): \delta=15.1$ $\left(\mathrm{NCH}_{2} \mathrm{CH}_{3}\right), 55.2\left(\mathrm{NCH}_{2} \mathrm{CH}_{3}\right), 108.9\left({ }^{2} J_{\mathrm{WC}}=25.0 \mathrm{~Hz}\right.$, $\left.\mathrm{C}_{\beta}\right), 121.9,130.9,143.3,143.8$ (py-C), $138.8\left(\mathrm{C}_{\gamma}\right), 164.8$ $\left({ }^{1} J_{\mathrm{WC}}=100.2 \mathrm{~Hz}, \mathrm{C}_{\alpha}\right), 198.6\left({ }^{1} J_{\mathrm{WC}}=125.2 \mathrm{~Hz}\right.$, cis $\left.-\mathrm{CO}\right)$, $202.8\left({ }^{1} J_{\mathrm{WC}}=132.8 \mathrm{~Hz}\right.$, trans-CO). MS (EI), $\mathrm{m} / z(\%)$ : $455(22)\left[\mathrm{M}^{+}\right], 399(34)\left[\mathrm{M}^{+}-2 \mathrm{CO}\right], 371(28)\left[\mathrm{M}^{+}\right.$ $3 \mathrm{CO}], 341(22)\left[\mathrm{M}^{+}-4 \mathrm{CO}\right], 315(72)\left[\mathrm{M}^{+}-5 \mathrm{CO}\right], 286$ (53) $\left[\mathrm{M}^{+}-5 \mathrm{CO}-\mathrm{Et}\right], 184(100)\left[\mathrm{W}^{+}\right]$. UV-vis: $\lambda_{\max }$ $(\log \varepsilon)$ [solvent]: $485 \mathrm{~nm}(4.303)$ [ $\mathrm{CHCl}_{3}$ ]; $465 \mathrm{~nm}$ (4.300) $\left[\mathrm{CH}_{2} \mathrm{Cl}_{2}\right] ; 426 \mathrm{~nm}$ (4.275) [DMF]. Anal. Found: C, 36.81; H, 1.97; N, 3.21. Calc. for $\mathrm{C}_{14} \mathrm{H}_{9} \mathrm{NO}_{5} \mathrm{~W}$ (455.08): C, 36.95; H, 1.99; N, 3.08\%.

\subsection{Pentacarbonyl [(e)-1,3-bis (dimethylamino)-3- phenylpropenylidene ]chromium (12)}

To a solution of $0.35 \mathrm{~g}(1 \mathrm{mmol})$ of pentacarbonyl(3dimethylamino-3-phenyl-1,2-propadienylidene)chromium (8a) in $30 \mathrm{ml}$ of dry $\mathrm{Et}_{2} \mathrm{O}$ was added $2 \mathrm{ml}$ of dimethylamine and was stirred at $20{ }^{\circ} \mathrm{C}$ until conversion was complete (indicated by TLC). Evaporation of the solvent and chromatographic workup $\left(-20{ }^{\circ} \mathrm{C}\right.$; pentane/ $\mathrm{CH}_{2} \mathrm{Cl}_{2}$ 1:1) gave $0.39 \mathrm{~g}(98 \%)$ of (12) as a yellow solid. M.p. $65-67^{\circ} \mathrm{C}$. IR (THF, $\left.\mathrm{cm}^{-1}\right): v(\mathrm{CO})=2047$ vw, 1919 vs, 1900 sh. ${ }^{1} \mathrm{H}-\mathrm{NMR}\left(400 \mathrm{MHz}, \mathrm{CDCl}_{3}\right): \delta=$ $2.59\left(\mathrm{~s}, 3 \mathrm{H}, \mathrm{NCH}_{3}\right), 2.66\left(\mathrm{~s}, 6 \mathrm{H}, \mathrm{NCH}_{3}\right), 3.36(\mathrm{~s}, 3 \mathrm{H}$, $\left.\mathrm{NCH}_{3}\right), 7.23\left(\mathrm{~d},{ }^{3} J_{\mathrm{HH}}=7.0 \mathrm{~Hz}, 2 \mathrm{H}, \mathrm{ArH}\right), 7.31(\mathrm{~m}, 3 \mathrm{H}$, $\mathrm{ArH}) .{ }^{13} \mathrm{C}-\mathrm{NMR}\left(100.5 \mathrm{MHz}, \mathrm{CDCl}_{3}\right): \delta=41.5$ $\left(\mathrm{NCH}_{3}\right), 45.7\left(\mathrm{NCH}_{3}\right), 50.1\left(\mathrm{NCH}_{3}\right), 118.3(\mathrm{C}=\mathrm{CH})$, 128.5, 128.6, 128.9, $137.4(\mathrm{ArC}), 142.4(C=\mathrm{CH}), 218.6$ (cis-CO), 224.0 (trans-CO). MS (EI), $\mathrm{m} / z$ (\%): 394 (2) $\left[\mathrm{M}^{+}\right], 366$ (4) $\left[\mathrm{M}^{+}-\mathrm{CO}\right], 310(12)\left[\mathrm{M}^{+}-3 \mathrm{CO}\right], 282(6)$ $\left[\mathrm{M}^{+}{ }_{-4 \mathrm{CO}}, 254(100)\left[\mathrm{M}^{+}-5 \mathrm{CO}\right]\right.$. UV-vis: $\lambda_{\max }(\log \varepsilon)$ [solvent]: $340 \mathrm{~nm}$ (4.063) $\left[\mathrm{CH}_{2} \mathrm{Cl}_{2}\right]$. Anal. Found: C, 54.70; H, 4.74; N, 7.03. Calc. for $\mathrm{C}_{18} \mathrm{H}_{18} \mathrm{~N}_{2} \mathrm{O}_{5} \mathrm{Cr}$ (394.35): C, 54.82; H, 4.60; N, 7.10\%.

\subsection{Tetracarbonyl(triphenylphosphane)(3-}

dimethylamino-3-phenyl-1,2-propadienylidene) chromium (15)

A solution of $0.35 \mathrm{~g}(1 \mathrm{mmol})$ of pentacarbonyl(3dimethylamino-3-phenyl-1,2-propadienylidene)chromium (8a) in $30 \mathrm{ml}$ of dry THF and $0.26 \mathrm{~g}(1 \mathrm{mmol})$ of triphenylphosphane were irradiated at $-20{ }^{\circ} \mathrm{C}$ for $2.5 \mathrm{~h}$ while passing a slow stream of argon through the solution. The solvent was removed and the oily deep violet residue was chromatographed at $-20{ }^{\circ} \mathrm{C}$ $\left(\mathrm{CH}_{2} \mathrm{Cl}_{2} / \mathrm{THF} 10: 1\right)$ to give $0.58 \mathrm{~g}(98 \%)$ of $\mathbf{1 5}$ as a violet solid. M.p. $88-89{ }^{\circ} \mathrm{C}$. IR (THF, $\left.\mathrm{cm}^{-1}\right): v(\mathrm{CO})=$ 2029 w, 1957 m, 1901 vs, 1875 m. ${ }^{1} \mathrm{H}-\mathrm{NMR}(400 \mathrm{MHz}$, $\left.\mathrm{CDCl}_{3}\right): \delta=3.20\left(\mathrm{~s}, 3 \mathrm{H}, \mathrm{NCH}_{3}\right), 3.36\left(\mathrm{~s}, 3 \mathrm{H}, \mathrm{NCH}_{3}\right)$, 7.18-7.44 (m, 20H, ArH). ${ }^{13} \mathrm{C}-\mathrm{NMR}(100.5 \mathrm{MHz}$, $\left.\mathrm{CDCl}_{3}\right): \delta=42.7\left(\mathrm{NCH}_{3}\right), 44.4\left(\mathrm{NCH}_{3}\right), 128.6(\mathrm{~d}$, $\left.{ }^{2} J_{\mathrm{PC}}=8.6 \mathrm{~Hz}, \mathrm{PArC}\right), 128.9\left(\mathrm{C}_{\beta}\right), 129.3\left(\mathrm{~d},{ }^{3} J_{\mathrm{PC}}=6.7\right.$ $\mathrm{Hz}, \mathrm{PArC}), 130.0\left(\mathrm{~d},{ }^{4} J_{\mathrm{PC}}=1.9 \mathrm{~Hz}, \mathrm{PArC}\right), 131.5,134.0$, 134.1, $136.0(\operatorname{ArC}), 138.2\left(\mathrm{~d},{ }^{1} J_{\mathrm{PC}}=30.8 \mathrm{~Hz}, \mathrm{PArC}\right)$, $151.8\left(\mathrm{~d},{ }^{3} J_{\mathrm{PC}}=2.0 \mathrm{~Hz}, \mathrm{C}_{\gamma}\right), 222.7\left(\mathrm{~d},{ }^{2} J_{\mathrm{PC}}=14.4 \mathrm{~Hz}\right.$, cis-CO), $229.5\left(\mathrm{~d},{ }^{2} J_{\mathrm{PC}}=3.8 \mathrm{~Hz}\right.$, cis-CO $\mathrm{CO}_{\text {trans }}$ to $\left.\mathrm{P}\right), 229.8$ $\left(\mathrm{d},{ }^{2} J_{\mathrm{PC}}=12.5 \mathrm{~Hz}\right.$, trans-CO), $236.8\left(\mathrm{~d},{ }^{2} J_{\mathrm{PC}}=21.2 \mathrm{~Hz}\right.$, $\left.\mathrm{C}_{\alpha}\right) .{ }^{31} \mathrm{P}-\mathrm{NMR}\left(162.0 \mathrm{MHz}, \mathrm{CDCl}_{3}\right): \delta=61.2$. $\mathrm{MS}(\mathrm{FAB}), \mathrm{m} / \mathrm{z}(\%): 584(8)\left[(\mathrm{M}+\mathrm{H})^{+}\right], 527(100)$ $\left[\mathrm{M}^{+}-2 \mathrm{CO}\right], 470(65)\left[\mathrm{M}^{+}-3 \mathrm{CO}+\mathrm{H}\right]$. UV-vis: $\lambda_{\max }$ $(\log \varepsilon)$ [solvent]: $581 \mathrm{~nm}$ (3.698) [pentane], $530 \mathrm{~nm}$ (4.207) $\left[\mathrm{CHCl}_{3}\right] ; 545 \mathrm{~nm}$ (4.133) $\left[\mathrm{CH}_{2} \mathrm{Cl}_{2}\right] ; 502 \mathrm{~nm}$ (4.103) [DMF]. Anal. Found: $\mathrm{C}, 68.01 ; \mathrm{H}, 4.92 ; \mathrm{N}$, 2.52. Calc. for $\mathrm{C}_{33} \mathrm{H}_{26} \mathrm{NO}_{4} \mathrm{PCr}$ (583.54): C, 67.92; $\mathrm{H}$, $4.49 ; \mathrm{N}, 2.40 \%$.

\subsection{X-ray structural analyses of $\mathbf{1 b}, 7 \boldsymbol{b}$, and $11 \boldsymbol{a}$}

Single crystals suitable for an X-ray structural analyses were obtained by slow diffusion of $n$-hexane into solutions of $\mathbf{1 b}, \mathbf{7 b}$, and $\mathbf{1 1 b}$ in $\mathrm{CH}_{2} \mathrm{Cl}_{2}$ at $-4{ }^{\circ} \mathrm{C}$. The measurements were performed with a crystal mounted on a glass fiber on a Siemens P4 diffractometer (graphite monochromator, $\mathrm{Mo}-\mathrm{K}_{\alpha}$ radiation, $\lambda=0.71073 \AA$ ). 
For the data collection the Wykoff technique was used. Semiempirical absorption correction $(\psi$ scan with 12 reflections) was performed. The structures were solved by direct methods using the SHELXTL-97 program package [31]. The positions of the hydrogen atoms were calculated by assuming ideal geometry, and their coordinates were refined together with those of the attached carbon atoms as 'riding model'. All other atoms were refined anisotropically.

\section{Supplementary material}

Crystallographic data for the structural analyses of complexes 1b, 7b and 11a have been deposited with the Cambridge Crystallographic Data Centre as supplementary publication no. CCDC-209313 (1b), CCDC-209314 (7b), and CCDC-209315 (11a). Copies of the data can be obtained free of charge on application to CCDC, 12 Union Road, Cambridge CB2 1EZ, UK (Fax: +441223-336033; e-mail: deposit@ccdc.cam.ac.uk or www: http://www.ccdc.cam.ac.uk).

\section{Acknowledgements}

Support of these investigations by the Fonds der Chemischen Industrie and the Wacker-Chemie $\mathrm{GmbH}$ (gift of chemicals) is gratefully acknowledged.

\section{References}

[1] Reviews: (a) M.I. Bruce, A, G. Swinger, Adv. Organomet. Chem. 22 (1983) 59. (b) M.I. Bruce, Chem. Rev. 91 (1991) 197. (c) S Doherty, J.F. Corrigan, A.J. Carty, E. Sappa, Adv. Organomet. Chem. 37 (1995) 39. (d) H.J. Werner, J. Chem. Soc. Chem. Commun. (1997) 903. (e) M.I. Bruce, Chem. Rev. 98 (1998) 2797. (f) D. Touchard, P.H. Dixneuf, Coord. Chem. Rev. (1998) 178. (g) V. Cadierno, M.P. Gamasa, J. Gimeno, Eur. J. Inorg. Chem. (2001) 571

[2] (a) H. Berke, G. Huttner, J.V. Seyerl, Z. Naturforsch. Sect. B 36 (1981) 1277

(b) H. Berke, P. Härter, G. Huttner, L. Zsolnai, Z. Naturforsch. Sect. B 36 (1981) 929;

(c) H. Berke, U. Großmann, G. Huttner, O. Rema, Z. Naturforsch. Sect. B 39 (1984) 1759.

[3] (a) H. Fischer, G. Roth, D. Reindl, C. Troll, J. Organomet. Chem. 454 (1993) 133;

(b) H. Fischer, D. Reindl, C. Troll, F. Leroux, J. Organomet. Chem. 490 (1995) 221;

(c) H. Fischer, G. Roth, J. Organomet. Chem. 507 (1996) 125;

(d) H. Fischer, D. Reindl, M. Gockel, C. Troll, Organometallics 17 (1998) 1393.

[4] (a) D.J. Bernad, M.A. Esteruelas, A.M. López, M. Oliván, E. Oñate, M.C. Puerta, P. Valerga, Organometallics 19 (2000) 4327; (b) V. Cadierno, S. Conejero, M.P. Gamasa, J. Gimeno, Organometallics 20 (2001) 3175;

(c) V. Cadierno, S. Conejero, M.P. Gamasa, J. Gimeno, Organometallics 21 (2002) 3716; (d) V. Cadierno, M.P. Gamasa, J. Gimeno, E. Pérez-Carreno, S. García-Granda, J. Organomet. Chem. 670 (2003) 75.

[5] S. Rigaut, J. Massue, D. Touchard, J.-L. Fillaut, S. Golhen, P.H. Dixneuf, Angew. Chem. 114 (2002) 4695.

[6] (a) M. Baya, M.L. Buil, M.A. Esteruelas, A.M. López, E. Onate, J.R. Rodríguez, Organometallics 21 (2002) 1841;

(b) E. Bustelo, M. Jiménez-Tenorio, K. Mereiter, M.C. Puerta, P. Valerga, Organometallics 21 (2002) 1903;

(c) M. Baya, M.L. Buil, M.A. Esteruelas, A.M. López, E. Oñate, J.R. Rodríguez, Organometallics 21 (2002) 1841.

[7] (a) A. Fürstner, M. Picquet, C. Bruneau, P.H. Dixneuf, Chem. Soc. Chem. Commun. (1998) 1315;

(b) M. Picquet, C. Bruneau, P.H. Dixneuf, Chem. Soc. Chem. Commun. (1998) 2249;

(c) A. Fürstner, A.F. Hill, M. Liebl, J.D.E.T. Wilton-Ely, Chem. Commun. (1999) 601;

(d) K.J. Harlow, A.F. Hill, J.D.E.T. Wilton-Ely, J. Chem. Soc. Dalton Trans. (1999) 285;

(e) A. Fürstner, M. Liebl, C.W. Lehmann, M. Picquet, R. Kunz, C. Bruneau, D. Touchard, P.H. Dixneuf, Chem. Eur. J. (2000) 1847 ;

(f) D. Sémeril, J. Le Notre, C. Bruneau, P.H. Dixneuf, A.F. Kolomiets, S.N. Osipov, New J. Chem. (2001) 16;

(g) R. Castarlenas, D. Sémeril, A.F. Noels, A. Demonceau, P.H. Dixneuf, J. Organomet. Chem. 663 (2002) 235;

(h) Y. Nishibayashi, Y. Inada, M. Hidai, S. Uemura, J. Am. Chem. Soc. 124 (2002) 7900;

(i) Y. Nishibayashi, I. Wakiji, M. Hidai, J. Am. Chem. Soc. 122 (2000) 11019;

(j) M. Saoud, A. Romerosa, M. Peruzzi, Organometallics 19 (2000) 4005;

(k) R. Akiyama, S. Kobayashi, Angew. Chem. 114 (2002) 2714; (l) R. Akiyama, S. Kobayashi, Angew. Chem. Int. Ed. 41 (2002) 2602;

(m) B.M. Trost, Acc. Chem. Res. 35 (2002) 695.

[8] (a) N. Re, A. Sgamellotti, C. Floriani, Organometallics 19 (2000) 1115;

(b) A. Marrone, N. Re, Organometallics 21 (2002) 3562.

[9] (a) B.M. Trost, J.A. Flygare, J. Am. Chem. Soc. 114 (1992) 5476; (b) S.J. Landon, P.M. Shulman, G.L. Geoffroy, J. Am. Chem. Soc. 107 (1985) 6739;

(c) R.H. Grubbs, S.J. Miller, G.C. Fu, Acc. Chem. Res. 28 (1995) 446 ;

(d) M. Schuster, S. Blechert, Angew. Chem. Int. Ed. Engl. 36 (1997) 2036;

(e) R.H. Grubbs, S. Chang, Tetrahedron 54 (1998) 4413;

(f) T. Suzuki, M. Tokunaga, Y. Wakatsuki, Tetrahedron Lett. 43 (2002) 7531.

[10] N. Mantovani, L. Marvelli, R. Rossi, V. Bertolasi, C. Bianchini, I. de la Rios, M. Peruzzini, Organometallics 21 (2002) 2382.

[11] (a) J.S. Schumm, D.L. Pearson, J.M. Tour, Angew. Chem. 106 (1994) 1445; Angew. Chem. Int. Ed. Engl. 33 (1994) 1360. Recent reviews: (b) W. Beck, B. Niemer, M. Wieser, Angew. Chem. 105 (1993) 969; Angew. Chem. Int. Ed. Engl. 32 (1993) 923. (c) H. Lang, Angew. Chem. 106 (1994) 569; Angew. Chem. Int. Ed. Engl. 33 (1994) 547. (d) U.H.F. Bunz, Angew. Chem. 108 (1996) 1047; Angew. Chem. Int. Ed. Engl. 35 (1996) 969.

[12] (a) D.W. Bruce, D. O'Hare (Eds.), Inorganic Materials, Wiley, Chichester, UK, 1992;

(b) Inorganic and Organometallic Polymers II: Advanced Materials and Intermediates, ACS Symposium Ser. 572, P. WisianNeilson, H.R. Wynne, (Eds.), American Chemical Society, Washington, DC (1994).

[13] (a) E.O. Fischer, H.J. Kalder, A. Frank, F.H. Köhler, G. Huttner, Angew. Chem. 88 (1976) 683;

(b) E.O. Fischer, H.J. Kalder, A. Frank, F.H. Köhler, G. Huttner, Angew. Chem. Int. Ed. Engl. 15 (1976) 623. 
[14] The synthesis of a di(t $t$-butyl)allenylidene complex of manganese, $\left[\mathrm{Cp}(\mathrm{CO})_{2} \mathrm{Mn}=\mathrm{C}=\mathrm{C}=\mathrm{C}\left(\mathrm{CMe}_{3}\right)_{2}\right], \quad$ from $\quad\left[\mathrm{Cp}(\mathrm{CO})_{2} \mathrm{Mn}(\mathrm{HC} \equiv\right.$ CCOOMe)], $t$-butylithium, and $\mathrm{HCl}$ was reported simultaneously: H. Berke, Angew. Chem. 88 (1976) 684; Angew. Chem. Int. Ed. Engl. 15 (1976) 624.

[15] R. Aumann, B. Jasper, R. Fröhlich, Organometallics 15 (1996) 3173

[16] (a) M. Duetsch, F. Stein, R. Lackmann, E. Pohl, R. Herbst-Irmer, A. de Meijere, Chem. Ber. 125 (1992) 2051;

(b) M. Duetsch, F. Stein, R. Lackmann, A. de Meijere, Synlett (1992) 324;

(c) M. Duetsch, F. Stein, E. Pohl, R. Herbst-Irmer, A. de Meijere, Organometallics 12 (1993) 2556;

(d) F. Stein, M. Duetsch, M. Noltemeyer, A. de Meijere, Synlett (1993) 486;

(e) H. Werner, T. Rappert, R. Wiedemann, J. Wolf, N. Mahr, Organometallics 13 (1994) 2721;

(f) R. Aumann, P. Hinterding, Chem. Ber. 126 (1993) 486;

(g) R. Aumann, Chem. Ber. 127 (1994) 725.

[17] (a) M.I. Bruce, P. Hinterding, P.J. Low, B.W. Skelton, A.H. White, J. Chem. Soc. Chem. Commun. (1996) 1009;

(b) M.I. Bruce, P. Hinterding, P.J. Low, B.W. Skelton, A.H White, J. Chem. Soc. Dalton Trans. (1998) 467;

(c) R.F. Winter, F.M. Hornung, Organometallics 16 (1997) 4248;

(d) R.F. Winter, Chem. Commun. (1998) 2209;

(e) R.F. Winter, K.-W. Klinkhammer, S. Záliš, Organometallics 20 (2001) 1317;

(f) R.F. Winter, S. Hartmann, S. Zálišs, K.-W. Klinkhammer, Dalton Trans. (2003) 2342.

[18] (a) A. Romero, D. Peron, P.H. Dixneuf, J. Chem. Soc. Chem. Commun. (1990) 1410;

(b) D. Peron, A. Romero, P.H. Dixneuf, Gazz. Chim. Ital. 124 (1994) 497; (c) D. Touchard, P. Haquette, A. Daridor, L. Toupet, P.H. Dixneuf, J. Am. Chem. Soc. 116 (1994) 11157;

(d) D. Peron, A. Romero, P.H. Dixneuf, Organometallics 14 (1995) 3319.

[19] G. Roth, H. Fischer, Organometallics 15 (1996) 1139.

[20] H. Fischer, D. Reindl, G. Roth, Z. Naturforsch. Sect. B 49 (1994) 1207.

[21] G. Dyker, S. Borowski, G. Henkel, A. Kellner, I. Dix, P.G. Jones, Tetrahedron Lett. 41 (2000) 8259.

[22] R. Aumann, K. Roths, M. Grehl, Synlett (1993) 669.

[23] B.W. Cook, R. Jackson, R.G.J. Miller, D.T. Thompson, J. Chem. Soc. (A) (1969) 25

[24] (a) V. Cadierno, M.P. Gamasa, J. Gimeno, E. Castra, J. Organomet. Chem. 474 (1994) C27;

(b) V. Cadierno, M.P. Gamasa, J. Gimeno, J. Borge, S. GarcíaGranade, J. Chem. Soc. Chem. Commun. (1994) 2495;

(c) V. Cadierno, M.P. Gamasa, J. Gimeno, M.C. López-Gonzáles, J. Borge, S. García-Granda, Organometallics 16 (1997) 4453;

(d) M.A. Esteruelas, A.V. Gómez, A.M. López, J. Modrego, E. Oñate, Organometallics 17 (1998) 5434;

(e) B. Weberndörfer, H. Werner, Dalton Trans. (2002) 1479.

[25] N.E. Kolobova, L.L. Ivanov, O.S. Zhvanko, O.M. Khitrova, Yu.M. Struchkov, J. Organomet. Chem. 265 (1984) 271.

[26] H. Fischer, D. Reindl, C. Troll, F. Leroux, J. Organomet. Chem. 490 (1995) 221.

[27] W.D. Crow, N.J. Leonard, J. Org. Chem. 30 (1965) 2660.

[28] C.B. Kanner, U.K. Pandit, Tetrahedron 38 (1982) 3597.

[29] K. Hartke, H.-D. Gerber, U. Roesrath, Liebigs Ann. Chem. (1991) 903.

[30] J. Schlegel, G. Maas, Synthesis (1999) 100.

[31] G.M. Sheldrick, SHELX-97, Programs for Crystal Structure Analysis, University of Göttingen, Göttingen (Germany), 1997. 